\title{
Innovative IoT Solutions and Wearable Sensing Systems for Monitoring Human Biophysical Parameters: A Review
}

\author{
Roberto De Fazio $^{1}$ (D), Massimo De Vittorio ${ }^{1,2}$ and Paolo Visconti ${ }^{1, *(D)}$ \\ 1 Department of Innovation Engineering, University of Salento, 73100 Lecce, LE, Italy; \\ roberto.defazio@unisalento.it (R.D.F.); massimo.devittorio@unisalento.it (M.D.V.) \\ 2 Center for Biomolecular Nanotechnologies, Italian Technology Institute IIT, 73010 Arnesano, LE, Italy \\ * Correspondence: paolo.visconti@unisalento.it; Tel.: +39-0832-297334
}

Citation: De Fazio, R.; De Vittorio, M.; Visconti, P. Innovative IoT Solutions and Wearable Sensing Systems for Monitoring Human Biophysical Parameters: A Review. Electronics 2021, 10, 1660. https:/ / doi.org/10.3390/electronics10141660

Academic Editors: Rashid Mehmood and Raed A. Abd-Alhameed

Received: 4 May 2021

Accepted: 5 July 2021

Published: 12 July 2021

Publisher's Note: MDPI stays neutral with regard to jurisdictional claims in published maps and institutional affiliations.

Copyright: (c) 2021 by the authors. Licensee MDPI, Basel, Switzerland. This article is an open access article distributed under the terms and conditions of the Creative Commons Attribution (CC BY) license (https:/ / creativecommons.org/licenses/by/ $4.0 /)$.

\begin{abstract}
Digital and information technologies are heavily pervading several aspects of human activities, improving our life quality. Health systems are undergoing a real technological revolution, radically changing how medical services are provided, thanks to the wide employment of the Internet of Things (IoT) platforms supporting advanced monitoring services and intelligent inferring systems. This paper reports, at first, a comprehensive overview of innovative sensing systems for monitoring biophysical and psychophysical parameters, all suitable for integration with wearable or portable accessories. Wearable devices represent a headstone on which the IoT-based healthcare platforms are based, providing capillary and real-time monitoring of patient's conditions. Besides, a survey of modern architectures and supported services by IoT platforms for health monitoring is presented, providing useful insights for developing future healthcare systems. All considered architectures employ wearable devices to gather patient parameters and share them with a cloud platform where they are processed to provide real-time feedback. The reported discussion highlights the structural differences between the discussed frameworks, from the point of view of network configuration, data management strategy, feedback modality, etc.
\end{abstract}

Keywords: advanced sensing; wearable devices; Internet of Things; biophysical parameters; cloud computing; fall detection algorithms; inertial sensors

\section{Introduction}

The rapid evolution of technology in recent decades has led to availability in the market of ICT (Information and Communications Technology) devices that feature increasingly cutting-edge performance, allowing for a significant improvement in daily activities [1-3]. Due to the COVID-19 emergency, a further boost occurred in the digitization process, encouraging the collaboration between medicine and technology to implement increasingly accurate health services and allowing a significant technological development in the health sector and the culture of prevention [4-6]. In this area, interest has grown to design new devices and systems constantly available and wearable, thanks to new solutions increasingly comfortable and smaller [7]. Internet of Medical Things (IoMT) platforms are revolutionizing the health systems, opening new frontiers in home medical care, allowing integrated, capillary, and precise monitoring of the patients' conditions, reduction of management costs, better diagnosis, earlier prevention, continuous tracking, and more rapid intervention [8-10]. These platforms aim to provide a secure connection to source devices for collecting data, processing them to extract helpful information, and securely storing them. Several technologies can be exploited and combined in the IoT (Internet of Things) platforms, such as advanced sensing, wearable devices, cloud and fog computing, and predictive and inferring techniques [11,12]. 
Wearable devices are powerful tools for supporting IoT systems because of their sensing, processing, and communication capability $[13,14]$. The term wearable devices cover a wide range of products integrated into clothing and accessories worn by the user and constantly connected to other intelligent electronic systems and the Internet network, allowing the detection, storage, and exchange of data in real-time and without human intervention $[15,16]$. Particularly, they allow detection of patients' vital parameters (e.g., heart rate (HR), oxygen saturation- $\left(\mathrm{SpO}_{2}\right)$, body temperature, blood pressure (BP), etc.) through advanced sensors positioned in direct contact with the skin, enabling several advantages such as continuous monitoring, reduced health costs and hospitalization times, and more straightforward prescription of preventive care, crucial for elderly people and infants $[17,18]$. Moreover, the development of advanced sensing devices for monitoring biophysical and environmental parameters, with features including small sizes, reduced power consumption, non-invasiveness [19], and flexibility [20,21], is fundamental for developing the next generation of wearable devices.

The main contributions of the proposed review work are:

- A comprehensive overview of recent advances in sensing technologies to monitor the biophysical parameters suitable for integration into wearable and portable devices; specifically, we focused on advanced techniques for monitoring HR, BP, respiration rate (RR), blood glucose level and others, in a non-invasive or even contactless way. In addition, wearable applications involving Galvanic Skin Response (GSR) measurements are discussed, a standard indicator of the physical or psychological conditions, for predicting epileptic seizures [22], diagnosing bipolar dysfunction [23], and detecting sleepiness [24]. We investigated the application of sweat sensors for rapid and non-invasive detection of toxins, drugs, hormones, alcohol, and glucose level [25].

- An overview of the IoT systems for health monitoring applications, focusing on the different IoT architectures and supported services. Analyzed systems make heavy use of wearable devices for high-resolution detection of the patient parameters, along with edge/fog computing to limit their requirements in terms of computational resources, power consumption, and energy autonomy [26]. Furthermore, cloud computing allows integrated management, and processing and storing of acquired data can be carried out. In this context, Artificial Intelligence (AI) and Machine Learning (ML) techniques can be integrated into their supported services to simplify the handling of a massive data amount and to infer useful information from them [27]. Finally, IoT frameworks for gait and life-quality monitoring are discussed, focusing on IoT systems for remote fall detection, based on wearable devices and ML techniques [28].

The rest of this review paper is arranged as follows: Section 2 reports an overview of the innovative sensing systems and methodologies to monitor biophysical parameters and psychological stress status. In Section 3, the architectures and services offered by IoT systems for health and life-quality monitoring are discussed.

\section{Overview of Innovative Sensing Systems and Algorithms for Monitoring Biophysical Parameters}

In the following section, we will first analyze the innovative methods and sensors for detecting biophysical parameters for monitoring human health as well as their applications in wearable devices.

In particular, several technologies and methodologies have been proposed in the scientific literature to monitor cardiovascular parameters, such as HR and BP, non-invasively. In [29], the authors propose a new technology called transdermal optical imaging (TOI) for measuring the BP using a video of a person's face, obtaining comparable accuracy to traditional blood pressure monitoring techniques (Figure 1). 


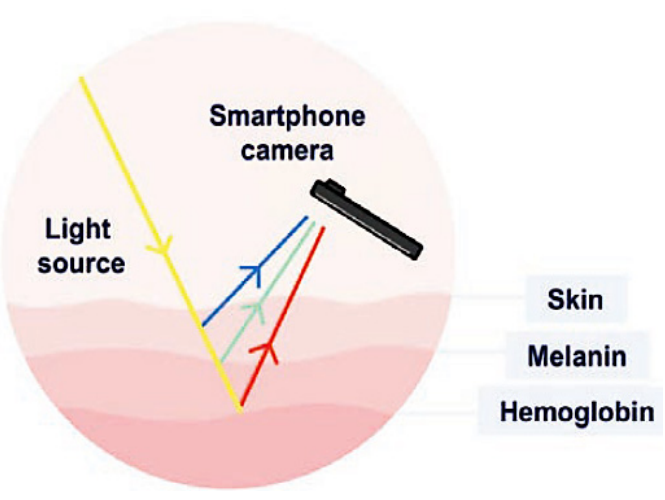

(a)
(1)

(4)

(5)

(6)

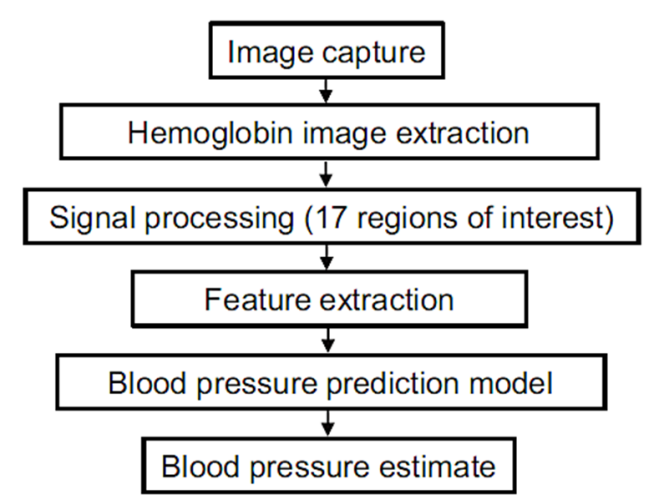

(b)

Figure 1. Graphical representation of TOI operation; (a) the light is re-emitted before being captured by the camera and moves beneath the skin surface. TOI technology, through the difference in re-emitted light between hemoglobin and melanin chromophores, uses the subtle changes in skin color to detect blood flow pulsation in the cardiovascular system; (b) operation sequence of TOI technique (1) image capture using a camera, (2) hemoglobin spatiotemporal images extraction, (3) hemoglobin signal processing, (4) feature extraction, (5) blood-pressure prediction trough model with advanced ML algorithms, and (6) blood pressure estimate [29].

They employed an advanced machine learning algorithm to create computational models that predict reference systolic, diastolic, and pulse pressure from the data related to facial blood flow. In this technique, the ambient light that penetrated the epidermis was used to reach the underlying tissue, with a part of it reflected outside. The smartphone's optical sensors were used to detect the re-emitted light and its small attenuations (Figure 1). Thus, information about hemoglobin and melanin can be extracted by analyzing the re-emitted frequency based on the differential absorption characteristics of these two lightabsorbing proteins. A bracelet was placed on the right arm at the approximate level of the heart (right atrium), thus permitting calibration of the continuous non-invasive arterial pressure (CNAP) reading for brachial BP (Figure 2).



Figure 2. Data collection setup used in [29].

Imaging techniques were used to extract and amplify the cyclic pulsations of blood within the human facial vascular system. The result was a spatio-temporal facial map of the TOI signal (Figure 3a). Then, the seventeen facial regions were identified, providing useful hemoglobin signals (Figure $3 \mathrm{~b}$ ). The TOI signal is then processed in the seventeen 
regions to determine whether it is representative of BP. The tests demonstrated that systolic and diastolic BPs are within $5 \pm 8 \mathrm{~mm} \mathrm{Hg}$ compared to the reference measurements.

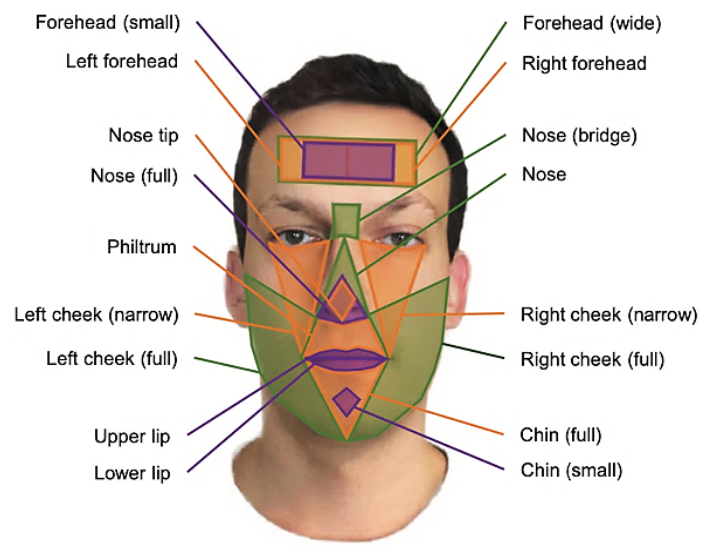

(a)

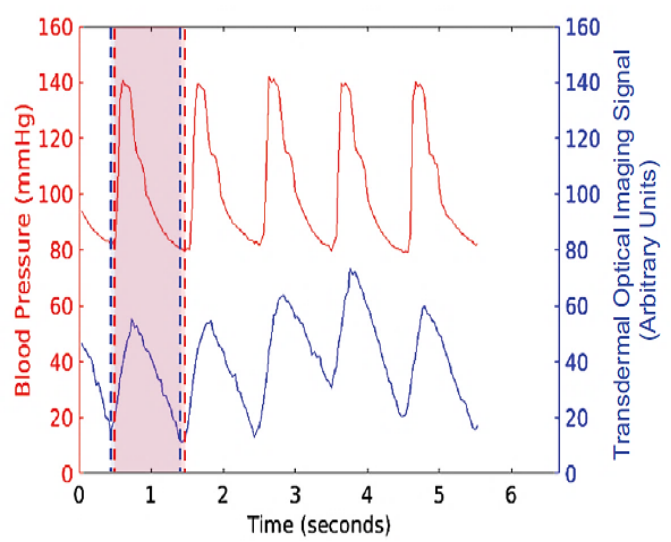

(b)

Figure 3. Regions of signal extraction and blood pressure signal; (a) demonstration of the seventeen regions of interest (forehead, nose, cheek, chin, lip, and philtrum); (b) recorded TOI signals from a person (raw signal from the green channel; presumed hemoglobin signal in the forehead region of interest) and reference blood-pressure signal (CNAP Monitor 500) exhibit temporally consistent oscillations and occur at $\approx 1$ per second [29].

In [30], the authors extend the oscillometric principle by developing a cuff-less BP monitoring device employing a smartphone to implement a real-time technique. The finger's user must be placed on the telephone cover, where two photoplethysmography (PPG) sensors and force transducers were integrated to measure the blood volume oscillations and applied pressure. In addition, the device prototype is integrated into a 3D-printed case, installed on the back of a mobile phone. The user can monitor the systolic, diastolic, and mean blood pressure on the brachial artery through a mobile application.

In [31], the authors developed a flexible self-powered pressure sensor (HSPS) tuned with a hierarchical elastomer (HEM) to achieve high sensitivity and a wide pressure range; the sensor could be suitable as a wearable cardiovascular healthcare device. The HSPS was constituted by HEM inserted between two ultra-thin PDMS layers. The HSPS obtained high sensitivity $\left(7.989 \mathrm{~V} \mathrm{kPa}^{-1}\right)$, a wide operative pressure range $(0.1-60 \mathrm{kPa})$, a fast response time $(40 \mathrm{~ms})$, and a high signal-to-noise ratio $(38 \mathrm{~dB})$ with high stability. Furthermore, the sensor successfully monitored arterial stiffness, blood pressure, and cardiac variability to assess cardiac and vascular conditions.

In [32], the authors introduced an innovative device for detecting pressure waves generated by the carotid artery in a non-invasive manner, using a pressure sensor enclosed in an air-filled housing. The ratios of volume and pressure of the casing in the initial position, without deformation to the deformed position, are obtained by exerting a constant force (Figure 4a,b). The signal acquisition was based on an analog MPXA6115A6U pressure sensor (NXP Semiconductors, Eindhoven, The Netherlands), placed inside the casing, which generates a voltage as a function of the internal pressure, acquired by an Arduino Uno board. Specifically, a polypropylene sheet with $0.125 \mathrm{~mm}$ thickness was modelled on the hemispherical structure, previously heated, and it is pushed through the hole to give it the desired dome shape (Figure 4c). Then, the housing is soldered to the PCB perimeter with an epoxy resin (Figure 4d). 


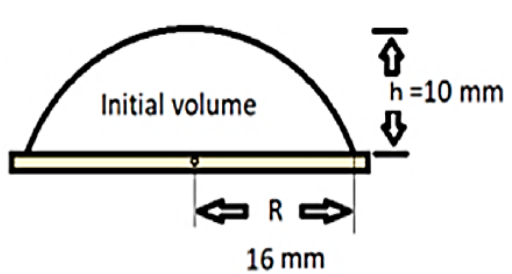

(a)

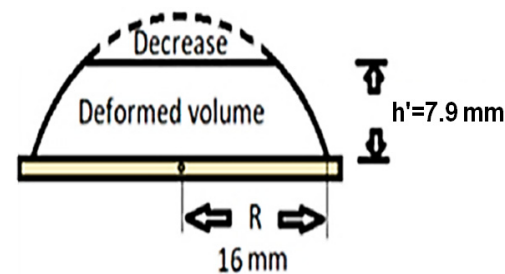

(b)

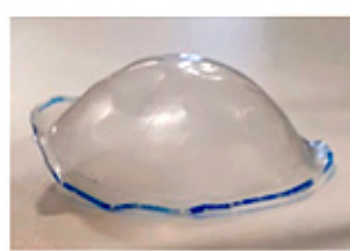

(c)

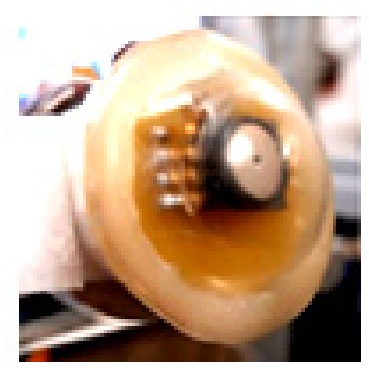

(d)

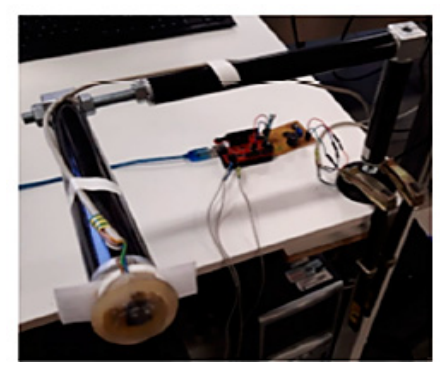

(e)

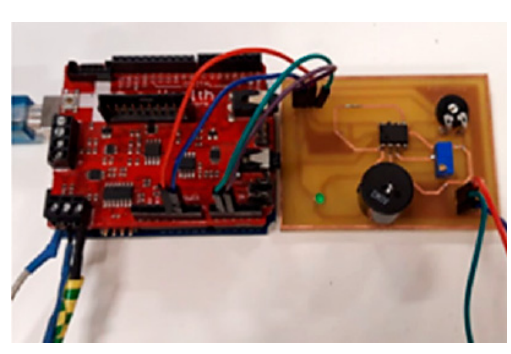

(f)

Figure 4. (a) Initial position without deformation; (b) deformed housing positioned on the artery; (c) dome housing; (d) housing glued on the PCB; (e) anchor system; (f) sound alarm system [32].

Since the internal pressure values increase due to manual force during the positioning or for its handling for the measurement, a fixed table anchor holder is realized to support the device (Figure 4e). Warnings are generated by a buzzer (Figure 4f), which warns when the pressure recorded by the sensor is within a minimal range from $108 \mathrm{kPa}$ to $109 \mathrm{kPa}$ (810.06-817.56 mmHg).

The holder was positioned and regulated before each test, during which the user approached the device until it touched the neck, as shown in Figure 5a. An algorithm was developed to identify diastolic points (minimum pressure signal), systolic points (maximum pressure signal), and dicrotic notches (aortic pressure transients). The employed algorithm, shown in Figure 5b, calculated the so-called R-points of the electrocardiogram (ECG) wave (systolic points corresponding to the heart contraction phase), thus obtaining the maximum pressure points of the blood pressure wave.

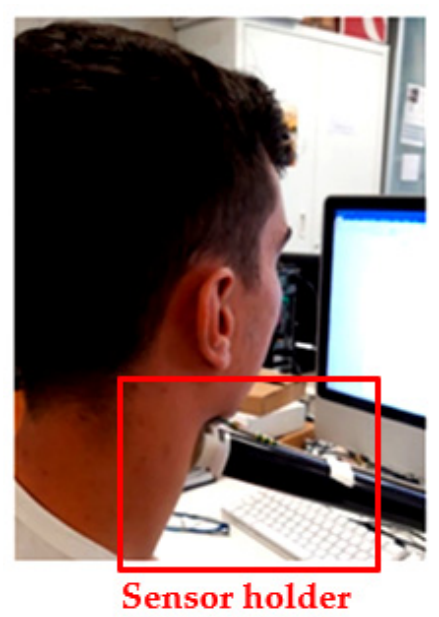

(a)
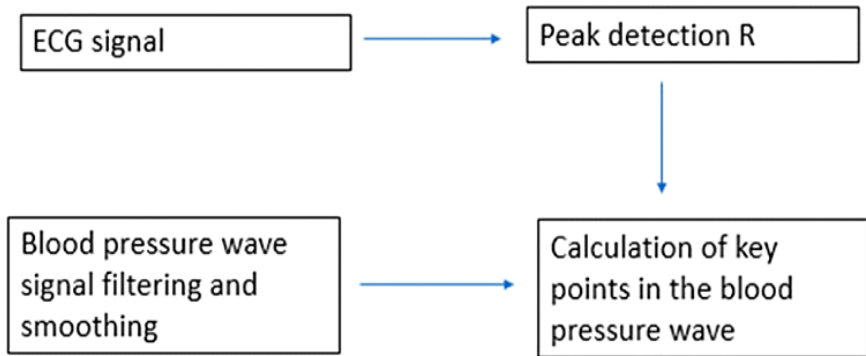

(b)

Figure 5. (a) Pressure wave measurement setup; (b) block diagram of the algorithm used for blood pressure [32]. 
Diabetes is undoubtedly one of the most diffused diseases affecting industrialized countries, highly influencing the lifestyle of those affected; therefore, quick and contact-less monitoring is essential to keep the disease under control. In [33], S. Haxha et al. developed a non-invasive glucose-monitoring system based on spectroscopy. The system included a Near Infra-Red (NIR) light source and a photodetector for measuring the luminous component transmitted by the sample; at first, a calibration process on a sample known thickness is carried out by placing it between the light source (NIR) and the detector (Figure 6). The sensor output was converted using the ADC (Analog to Digital Converter) module; the data were then filtered (for removing noise) and the concentration values were calculated using a data processing algorithm. Then, the obtained value was taken as a reference to compare future datasets, and a multiplier extraction function is implemented. Besides, they extracted the relationship between the sensor output voltage and glucose concentration (Figure 7). The proposed glucose monitoring system showed excellent cost reduction, maintenance perspectives, and good accuracy.

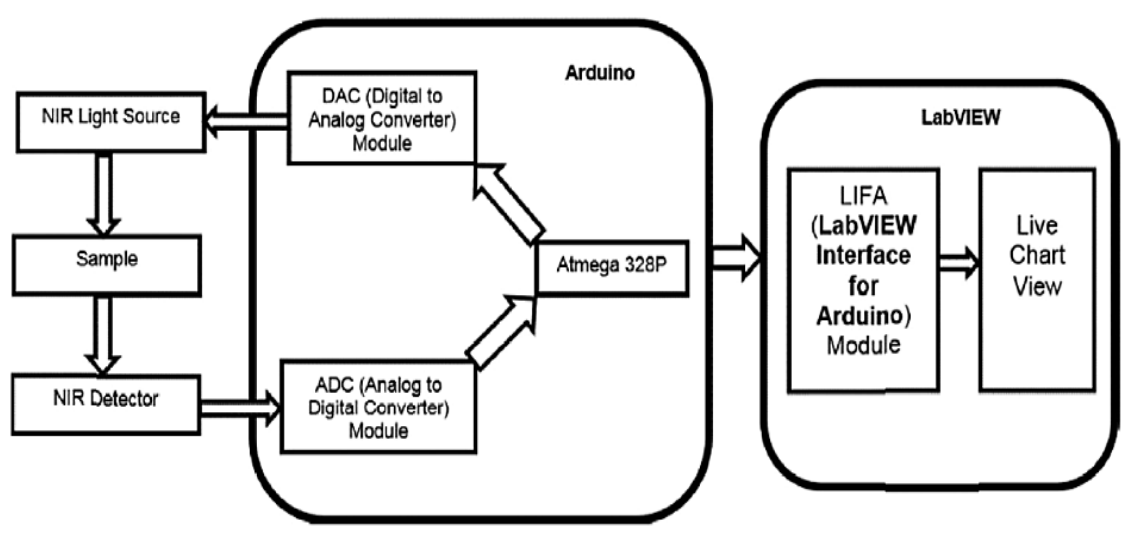

Figure 6. Block diagram of the non-invasive glucose monitoring system proposed in [33].

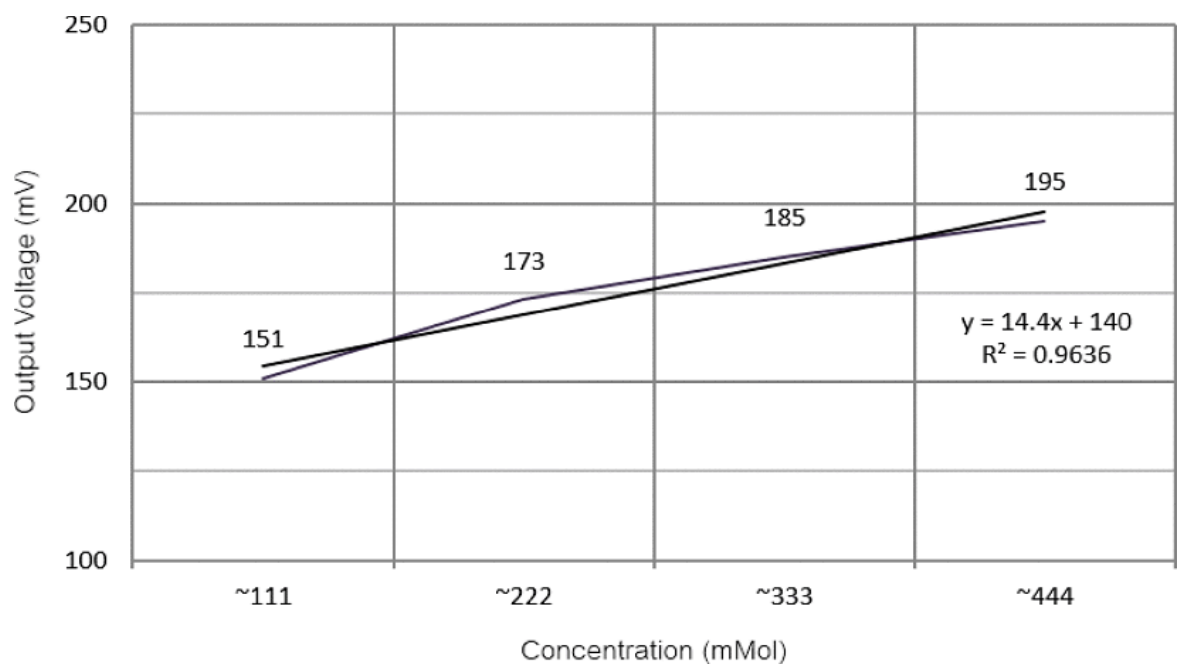

Figure 7. Experimental results for different glucose aqueous solution concentrations [33].

Figure 8 illustrates the 3D model of the developed non-invasive glucose monitoring system. The obtained results demonstrated that the integration of NIR technology in the biomedical field promises future success, especially in optical spectroscopy for noninvasive, real-time, and continuous monitoring of glucose concentration. 


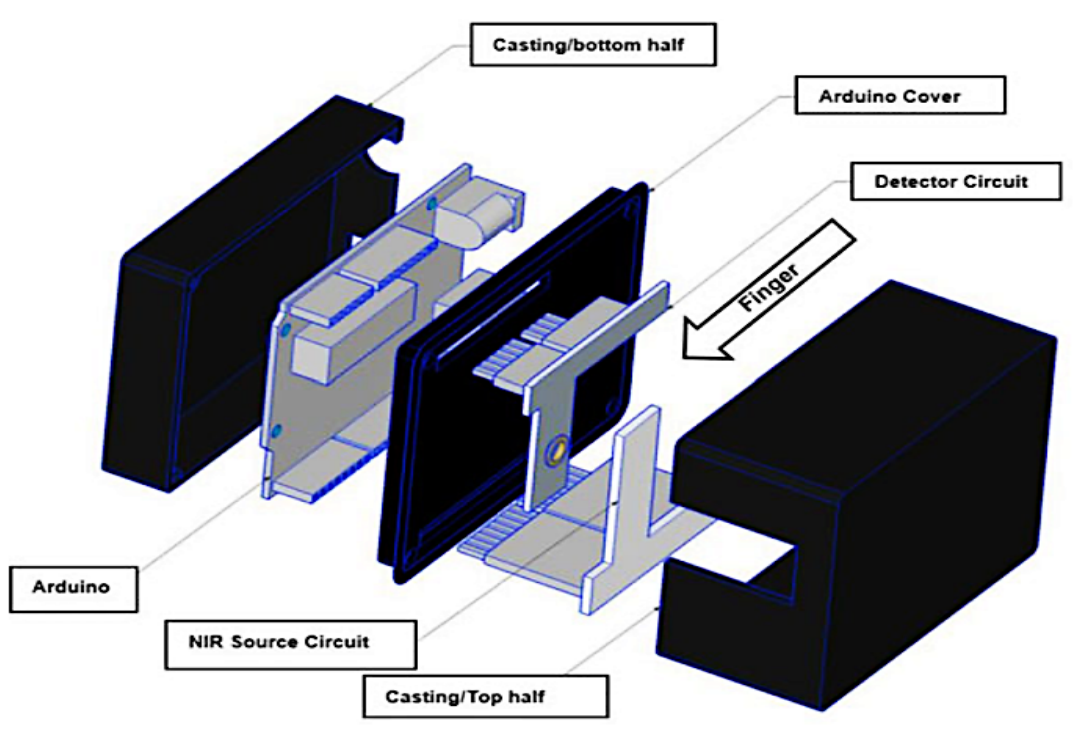

Figure 8. 3D view of the non-invasive glucose monitoring system, proposed in [33], with the main components highlighted.

Furthermore, in [34], the authors proposed a prediction method of blood glucose based on photoacoustic spectroscopy (PAS). This uses a laser beam in the middle infrared interacting with the skin, producing a thermal expansion and generating an acoustic wave, altered by the sample absorption coefficient and the physical properties of the propagation medium, including the coefficient of thermal expansion and velocity. Even if the depth of penetration of mid-infrared light into the skin $(<100 \mu \mathrm{m})$ was low, the interstitial fluids in the granular layer and the spinous layer were reached by mid-infrared light under the corneum stratum, i.e., the outermost layer of the skin with a thickness of $\sim 15-20 \mu \mathrm{m}$. The method showed an inherent variability due to the skin secretions interfering with mid-infrared light, namely the sebum and sweat. To overcome these problems, a position scanning photoacoustic spectroscopy system has been developed. A microphone equipped with a phase-sensitive amplifier and filters is employed to acquire the photoacoustic signal. The laser beam was focused up to $90 \mu \mathrm{m}$ diameter, close to the focus at $50.4 \mathrm{~mm}$. The skin's acoustic pulse produced by the laser absorption was coupled to the photoacoustic cell, in turn, matched with the laser repetition frequency $(47.5 \mathrm{kHz})$. In vivo tests indicated that measurements on the no secretion position produced a better accuracy than that on the secretion position.

In [35], the authors designed a wearable blood glucose (BG) sensor with an excellent cost-performance ratio, enabling continuous blood glucose (CGM) monitoring. This sensor acquires optical signal using the combined near-infrared-visible (NIR-Vis) spectroscopy, identifying the continuous components of the arterial blood volume pulse and blood glucose (BG) concentration changes.

Numerous experiments have been carried out, demonstrating the device's reliability and accuracy. At first, the device was compared with other commercially derived devices to estimate the HR; subsequently, the comparison was made on BG. As aforementioned, the device uses a combined NIR-Vis analysis; many LEDs were tested to reach a depth resolution of the blood pulsation under the wrist tissue. A NIR frequency beam can penetrate through the skin and reach the arteries; in contrast, visible light only reaches the capillaries and arterioles region, located in dermis tissue, as shown in Figure 9a. Multivariate analysis was applied, collecting reflected light at different optical wavelengths, extracted by a proper signal system. 


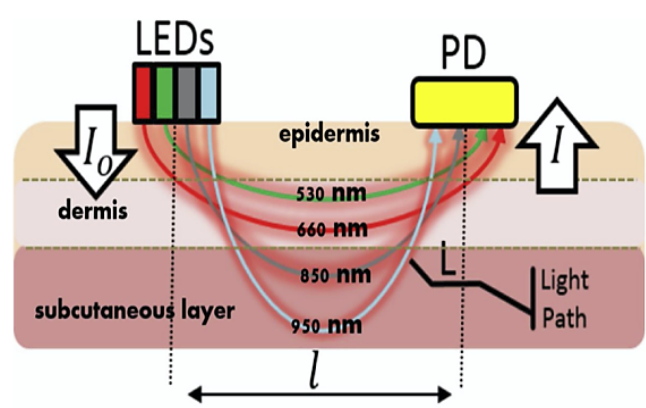

(a)

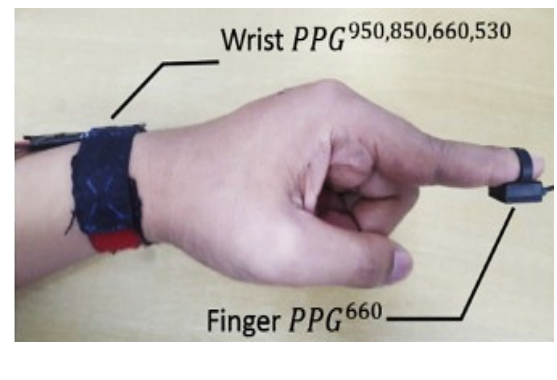

(b)

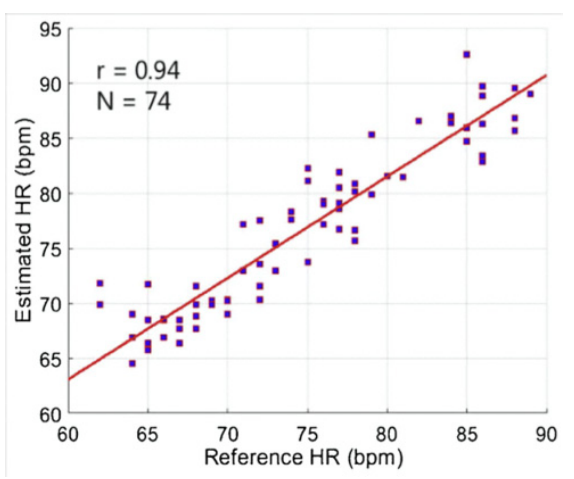

(c)

Figure 9. PPG-based device used for the measurement of multiple optical wavelengths; (a) sensor structure, (b) the biosensor placed on the wrist of the submissive, a commercial PPG placed on the finger, (c) correlation diagram of the estimated heart rate from the proposed structure with the commercial device [35] (Reprinted with permission from ref. [35]. Copyright 2021 Elsevier).

The sensors were placed on the cuff backside or finger, in contact with the skin, and located in correspondence to interosseous arteries (Figure 9b). The authors used PLS (Partial Least Squares) for the BG calibration and estimation model and compared with other methods. Four optical wavelengths were investigated, and the correspondent PPG $950,850,660,530$ were measured. Finally, the authors found out that the NIR-Vis featurebased model overcomes the other model, obtaining a Standard Error of Prediction (SEP) lower than $6.16 \mathrm{mg} / \mathrm{dl}$ for a correlation coefficient $\left(R_{p}\right)$ higher than 0.86 (Figure 9c).

The Galvanic Skin Response (GSR) is the variation of the skin resistance due to the change in sweat secretion by the sweat glands (i.e., eccrine and apocrine), ascribable to different emotive or physiological causes. This quantity is correlated, for instance, to stress or pain conditions, as well as emotional states. A GSR sensor detects the skin conductance induced by a reaction not controlled by the user [36-38]. A common trend is the integration of GSR sensors inside wearable devices, allowing, through IoT platforms, remote detection of the pain or fear related to body unbalance and falls $[39,40]$ or stress due to the worsening of a previous pathology [41-43]. In [39], the authors proposed a wearable device to detect inertial properties and acceleration, through inertial measurement units (IMUs), along with values related to GSR, HR and BP. The GSR represents an indicator of surprising events or fear of falling due to the rapid response of the sympathetic system. The acquired data are fused and analyzed with a pattern algorithm to detect the dangerous event and provide immediate warning alarm.

In [44], the authors developed a GSR detection technique for interpreting electrodermal activity (EDA) records. Such applications are useful for both outpatient and healthcare applications, generating a new feature extraction method for GSR signals using the SparsEDA. The core of the research was a new scheme based on a sparse non-negative deconvolution of the observed GSR signals. The GSR signal $(\mathrm{s}(\mathrm{t})$ ) was the sum of two components, namely the tonic component $\mathrm{s}_{1}(\mathrm{t})$, called Skin Conductance Level (SCL) characterized by slow changing, and the phasic component $\mathrm{s}_{\mathrm{p}}(\mathrm{t})$, called Skin Conductance Response (SCR), with high-frequency components. The SCR can be expressed as the convolution between a sudomotor SNS innervation, $d_{p}(t)$, the non-negative unknown sparse driver causing the observable skin conductance response, and the driver, $r(t)$, that triggers the response. Finally, GSR signals are expressed as:

$$
\mathrm{s}(\mathrm{t})=\mathrm{s}_{\mathrm{p}}(\mathrm{t})+\mathrm{s}_{\mathrm{l}}(\mathrm{t})=\mathrm{d}_{\mathrm{p}}(\mathrm{t}) \times \mathrm{r}(\mathrm{t})+\mathrm{s}_{\mathrm{l}}(\mathrm{t}),
$$

The discrete-time transformation of the $\mathrm{s}(\mathrm{t})$ signal into a finite time interval can be expressed in matrix form: 


$$
\mathrm{s}[\mathrm{n}]=\mathrm{s}_{\mathrm{l}}[\mathrm{n}]+\mathrm{d}_{\mathrm{p}}[\mathrm{n}] \times \mathrm{r}[\mathrm{n}]+\mathrm{w}[\mathrm{n}],
$$

The main objective was to determine both sequence $d_{p}[n]$ and $s[n]$ when $r[n]$ (and thus $\mathrm{R}$ matrix) is unknown. The SparsEDA algorithm enables fast, adequate, and automated processing to determine the GSR components (SCL and SCR) with great EDA records. The algorithm has been tested on a database of 100 GSR records obtained from 100 different patients, confirming its good performance in terms of MSE-Mean Square Error- $-48.50 \mathrm{~dB})$ and processing time (1-2 orders of magnitude lower than existing techniques such as CDA Ledalab and cvxEDA).

In [45], the author described the design of a portable GSR for pain sensors to measure the changes of the skin's electrical resistance to determine the autonomic nerve responses correlated to sweating. The prototype measures skin conductivity and activity induced by light blows to the arms (Figure 10a-c). An Arduino Uno microcontroller acquires and processes the electrophysical signals for obtaining the interest information, sent to a PC using an HC-05 Bluetooth module and displayed via a serial plotter. The employed exosomatic method uses either direct current, called DC-exosomatic, or alternating current, AC-exosomatic. Figure 10a shows the simplified conditioning circuit to measure the skin resistance (SR); SC represents the resistance between two skin zones, where two conductive electrodes are placed. The resulting signal is then amplified by a specific factor depending on the monitored subject.

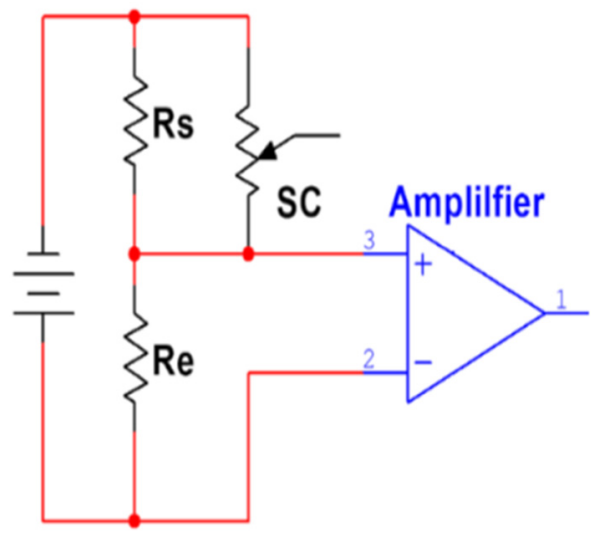

(a)

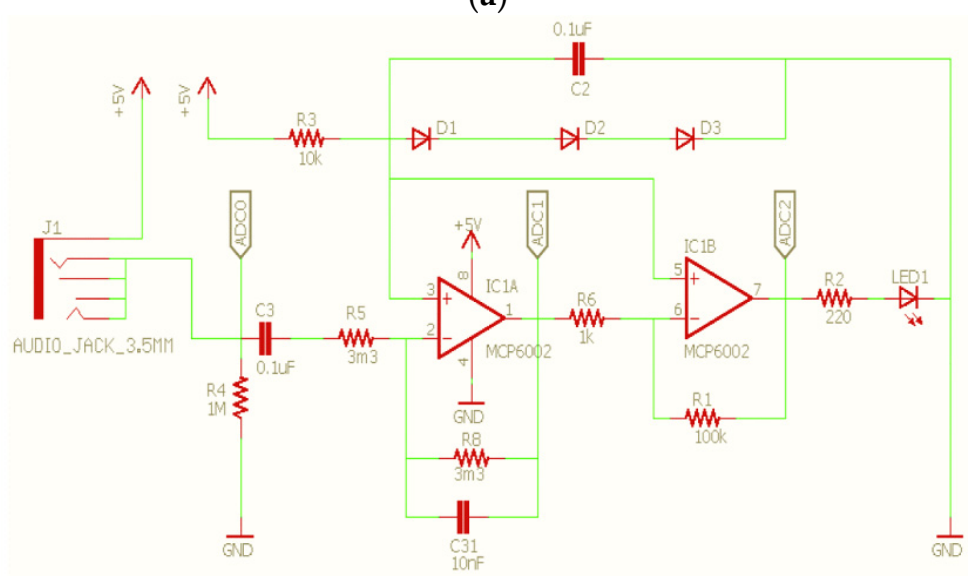

(b)

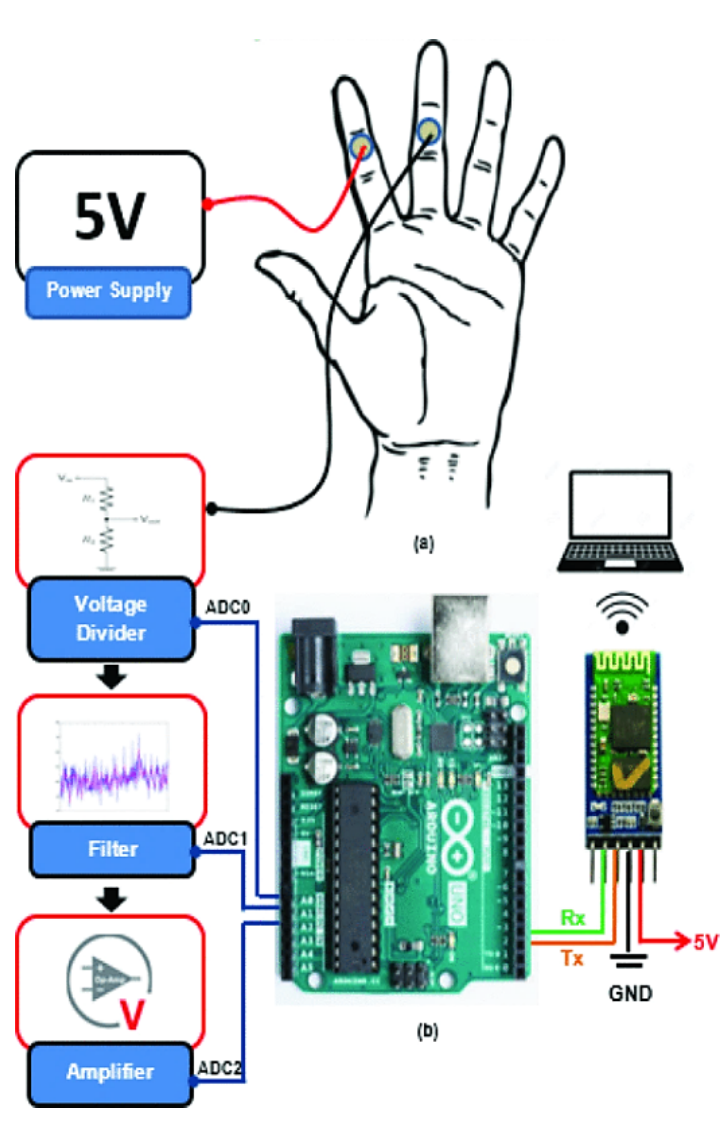

(c)

Figure 10. (a) Skin conductance measurement circuit using the exosomatic method; (b) scheme of the galvanic skin response circuit; (c) electrode positioning on the hand (up) and block diagram of typical galvanic skin response (down) [45] (Reprinted with permission from ref. [45]. Copyright 2021 IEEE). 
The conditioning section is shown in Figure 10b, divided into three stages, each with its functionality. Each stage has an output voltage, represented by the probes V1 (analog input ADC0), V2 (ADC1), and V0 (ADC2). The detected current is limited to $10 \mu \mathrm{A} / \mathrm{cm}^{2}$ to avoid damage to the sweat gland ducts. The processing determined the different applied stimuli using different readings obtained from the device, with a total elaboration time of 4 min. Figure 11 shows the galvanic skin responses to the applied stimuli.



Figure 11. Temporal trend of the galvanic skin response: (a) Initial condition; (b) first bump; (c) recovery state before the second blow; (d) second bump [45] (Reprinted with permission from ref. [45]. Copyright 2021 IEEE).

In [46], the authors propose a rule-based algorithm based on the galvanic skin response and skin temperature, with a very large correspondence with the empirical evidence and integrated with expert knowledge, enabling transferability to the real world.

GSR and Skin Temperature (ST) data were recorded through a wearable non-invasive bracelet called "Empatica E4", designed for research and clinical purposes. The E4 bracelet integrates several sensors to measure Blood Volume Pulse (BVP), GSR, Inter-Beat Interval (IBI), and ST in real-time. The eDiary application recorded GSR and ST data and perceived stress and emotion levels, creating a database containing physiological signals with a system timestamp for all measurements; the data can be geolocalized using a global satellite system (GNSS) integrated into the smartphone. Some rules were defined for induction during the laboratory experiment; then, various weights were assigned to the rules based on the hypothesis that each rule had different importance for stress detection. Finally, a ternary score was calculated in the multi-criteria decision-making process. The algorithm can detect, at most, $90 \%$ of induced MOS and $77 \%$ on average; also, induced MOSs, not considered as stress, have been detected.

In [47], the authors proposed a smart ring (called SensoRing) for continuous monitoring of EDA, HR, ST, and activity level (Figure 12). The SensoRing included three main sections: (1) The hardware section for collecting the signals from sensors; (2) the wireless communication protocol; and (3) the processing and transformation software. The acquired parameters were wirelessly transmitted to a processing platform, including custom made hardware architecture, signal pre-processing software, power management strategy, and seamless connectivity. In addition, the system was equipped with a graphical user interface (GUI), allowing the integration of different signal processing algorithms. The 3D model of the SensorRing prototype was obtained using the software SolidWorks (Figure 13). 


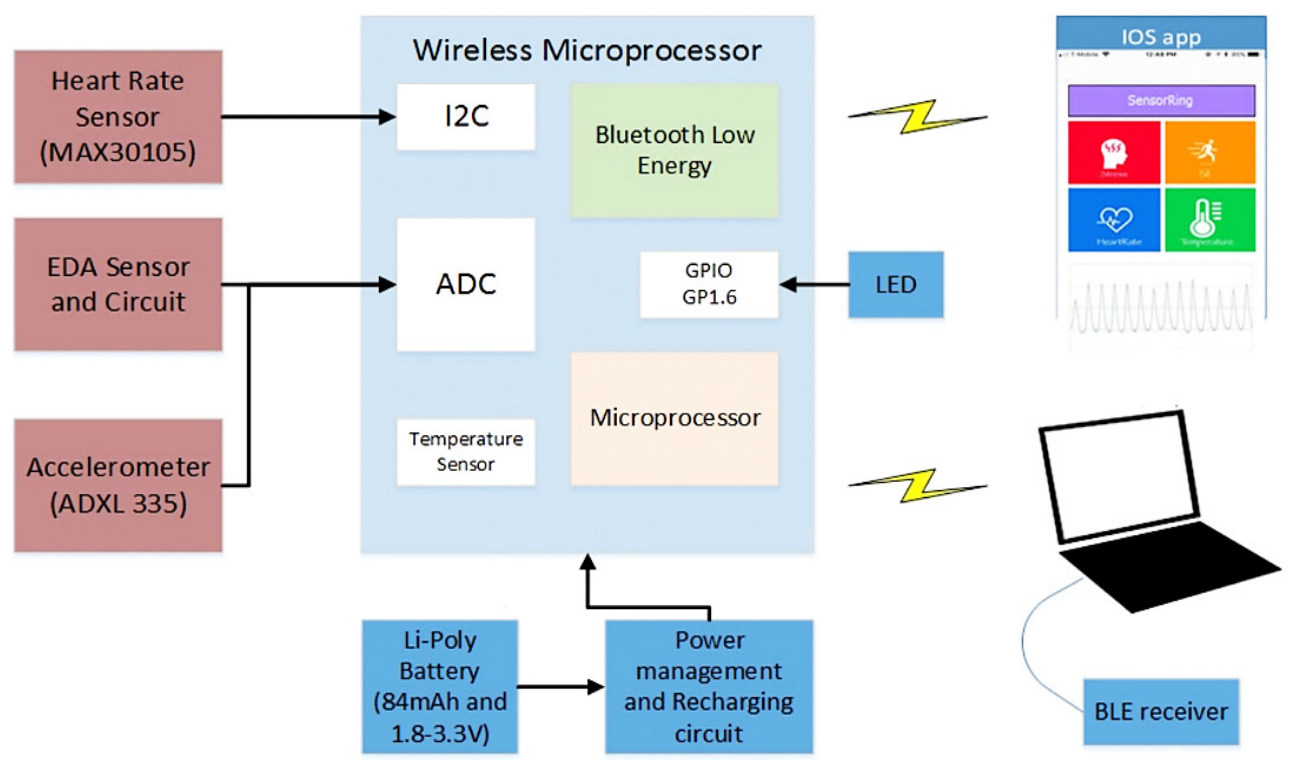

Figure 12. Sensor architecture in SensoRing proposed in [47] (Reprinted with permission from ref. [47]. Copyright 2021 IEEE).



Figure 13. Structure of the wearable system proposed in [47]: 3D model of SensorRing (left side) and disassembled device with support and cover (right side of the image) (Reprinted with permission from ref. [47]. Copyright 2021 IEEE).

In [48], a wearable system based on time-domain reflectometry (TDR) was introduced for determining skin hydration due to the relationship between dielectric properties and the water content of biological tissues. The TDR measurement relied on an electro-magnetic solicitation, propagating along the SE-sensor, in contact with the skin. The reflection coefficient $\rho(t)$ expresses the impedance changes, defined by the ratio between the amplitude of the reflected, $v_{\text {ref }}(t)$, and incident signals, $v_{\text {inc }}(t)$; this quantity was related to the material dielectric properties in which the TDR signal propagates.

$$
\rho(t)=\frac{v_{\text {ref }}(t)}{v_{\text {inc }}(t)}
$$

where $-1 \leq \rho(t) \leq 1$, and $d_{a p p}$ was related to the material dielectric properties.

$$
\mathrm{d}_{\mathrm{app}} \cong \mathrm{d} \times \sqrt{\varepsilon_{\mathrm{r}}}
$$

where $\mathrm{d}$ was the physical distance travelled by the propagating signal, and $\varepsilon_{\mathrm{r}}$ is the relative dielectric permittivity of the material on which the SE is placed. The SE element was constituted by a $13 \mathrm{~cm}$-long tri-filar ground-source-ground (GSG) planar structure, with fingers characterized by a width of $2 \mathrm{~mm}$ with a spacing of $1 \mathrm{~mm}$ (Figure 14). 




Figure 14. The schematization of the SE planar tri-filar to determine skin hydration [48].

The TDR responses were determined using a saline solution with different sugar concentrations (Figure 15).

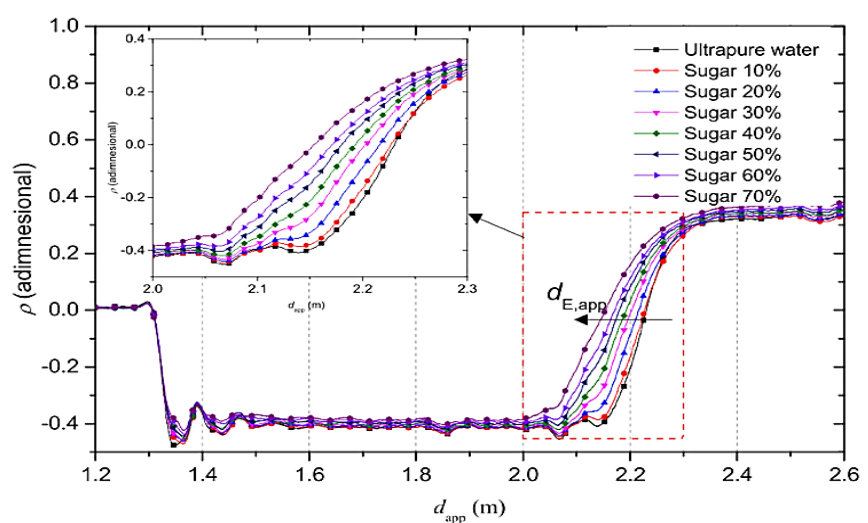

(a)

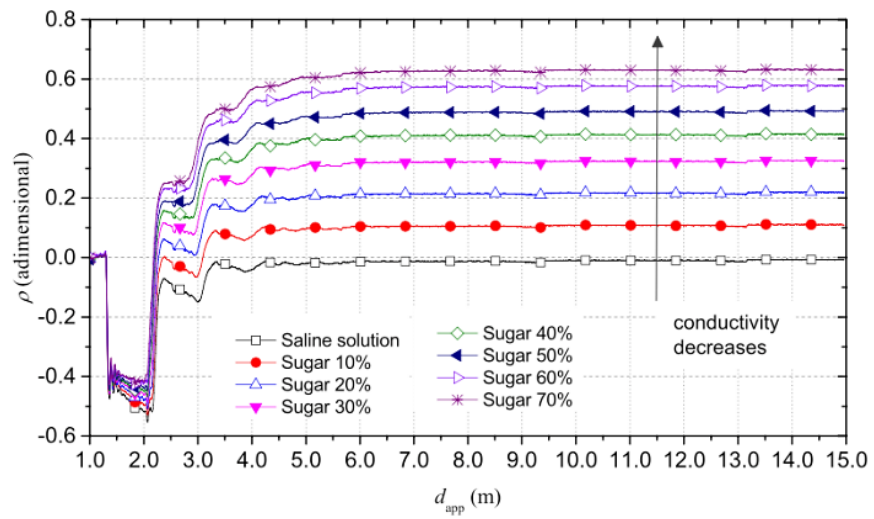

(b)

Figure 15. TDR reflectogram, (a) for ultra-pure water and (b) saline solutions with different sugar concentrations [48].

Measurements have been performed with SE fixed on the inner surface of the forearm by using a smartphone armband, as depicted in Figure 16a,b. The experimental measurements carried out to test the proposed solution are the following:

1. Forearm in dry condition;

2. Forearm with applied a skin lotion to increase the hydration level;

3. Forearm wet with water;

4. Forearm dried to remove lotion and water residues.

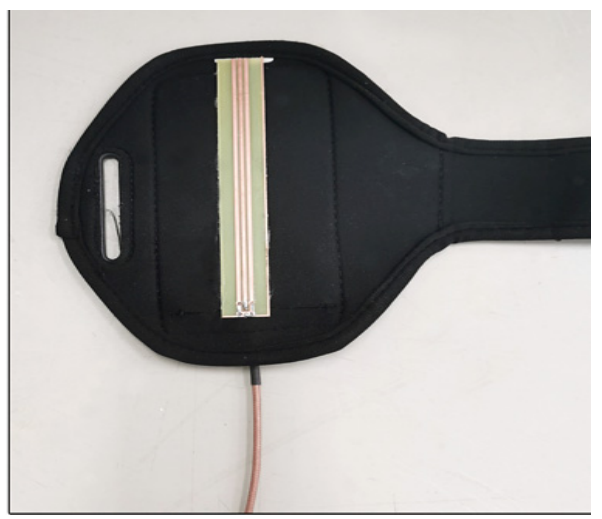

(a)

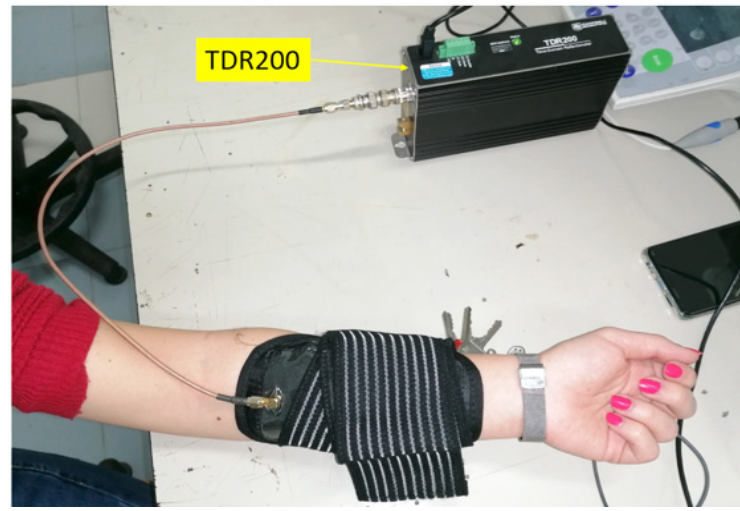

(b)

Figure 16. (a) SE attached to the inner surface of a smartphone bracelet running; (b) experimental setup for measuring human skin hydration [48].

The reflectograms for the four conditions previously mentioned are reported in Figure 17. 


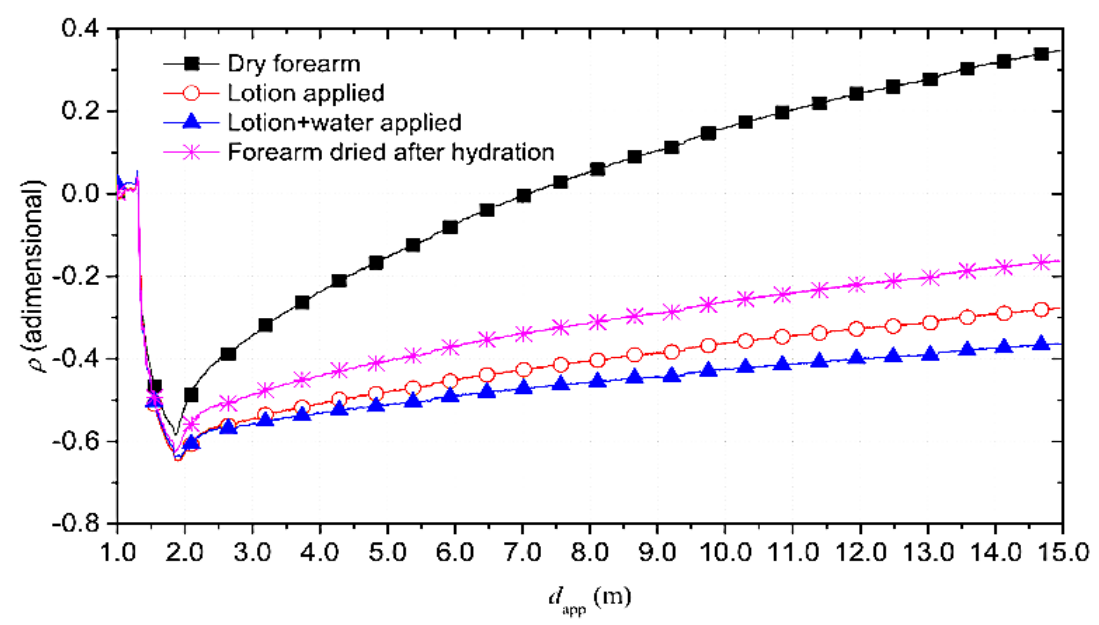

Figure 17. TDR reflectograms obtained in the four conditions described above [48].

Several sweat detection methods were proposed in the literature, suitable for wearable devices, including absorbent sensors, super-hydrophobic/hydrophilic surfaces, sweat guides, and epidermal microfluidic systems. The common sweat detection methods are:

- Whole-body washdown technique: It is the main technique to determine electrolyte loss since the outflow sweat is collected, and evaporative sweating is undisturbed;

- Patches: Consisting of a hydrophilic and porous structure placed between the skin and an external membrane. It is applied to the forearm, thigh, back, and calf;

- Polymer bags/films: A polyethene sheet with a thin layer of petroleum jelly and paraffin oil on the skin is used as an anaerobic sweat collector to gather large sweat volumes with less contamination and evaporative water loss;

- Macroducts: Plastic capsule placed on the skin with spiral plastic tube collecting sweat, avoiding sweat loss, contamination, and potential hydromeiosis.

Using in situ sampling techniques, the sensors perform real-time measurements in fresh sweat instead of the mixture of new and old sweat for autonomous and continuous detection (Figure 18).

Cell substrate pores improved the flow of sweat accumulated under the sensor, improving perspiration (Figure 18a) [49]. Figure 18b shows a "panda-like" tattoo platform including different hydrogels on the electrodes for efficient sampling and storage of biofluids [50]. Instead, Figure 18c illustrates a wireless extensible sensor for sweat detection on the skin's surface, consisting of an inductive coil and a planar capacitor [51]. Figure 18d shows a thin rayon pad between the skin and the sensor array, which completely absorbed sweat [52]. In Figure 18e, an ultra-thin and stretchable, and miniaturized patch is depicted, applicable as a disposable strip [53].

Furthermore, a hydrophobic/super-hydrophilic fabric was developed by modifying a commercial Janus based on polyester/nitrocellulose (PE/NC) with asymmetrical and regularly hydrophilic tapered micropore arrays for directional sweat transport (Figure 19a) [54]. On the band, the sweat droplets were repelled by the super-hydrophobic silica coating and collected on super-hydrophilic wells guided by the extreme wettability gradient with minimal lateral diffusion (Figure 19b) [55]. 

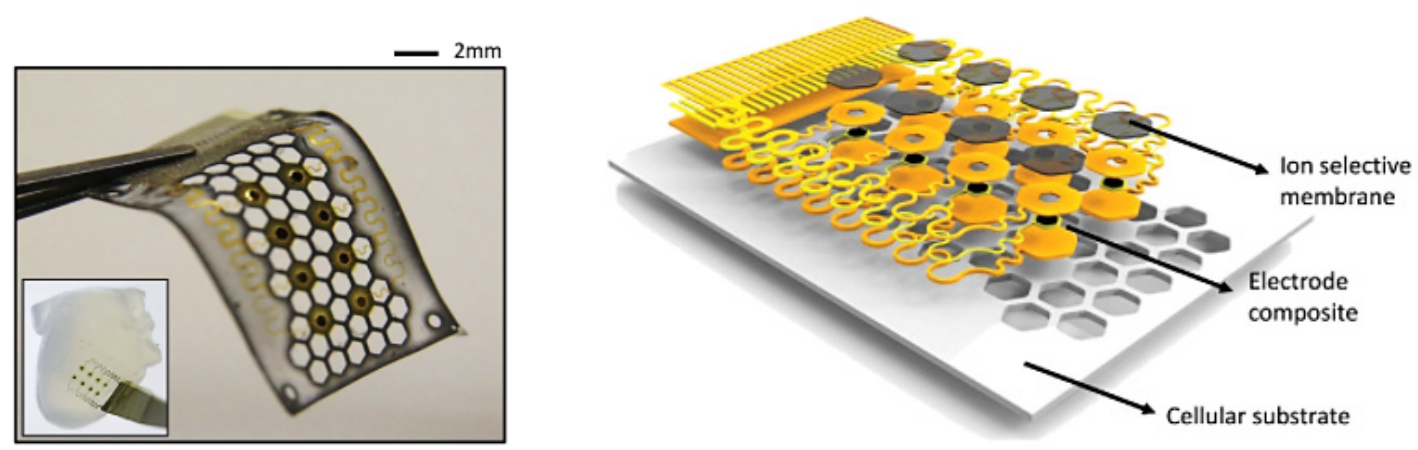

(a)
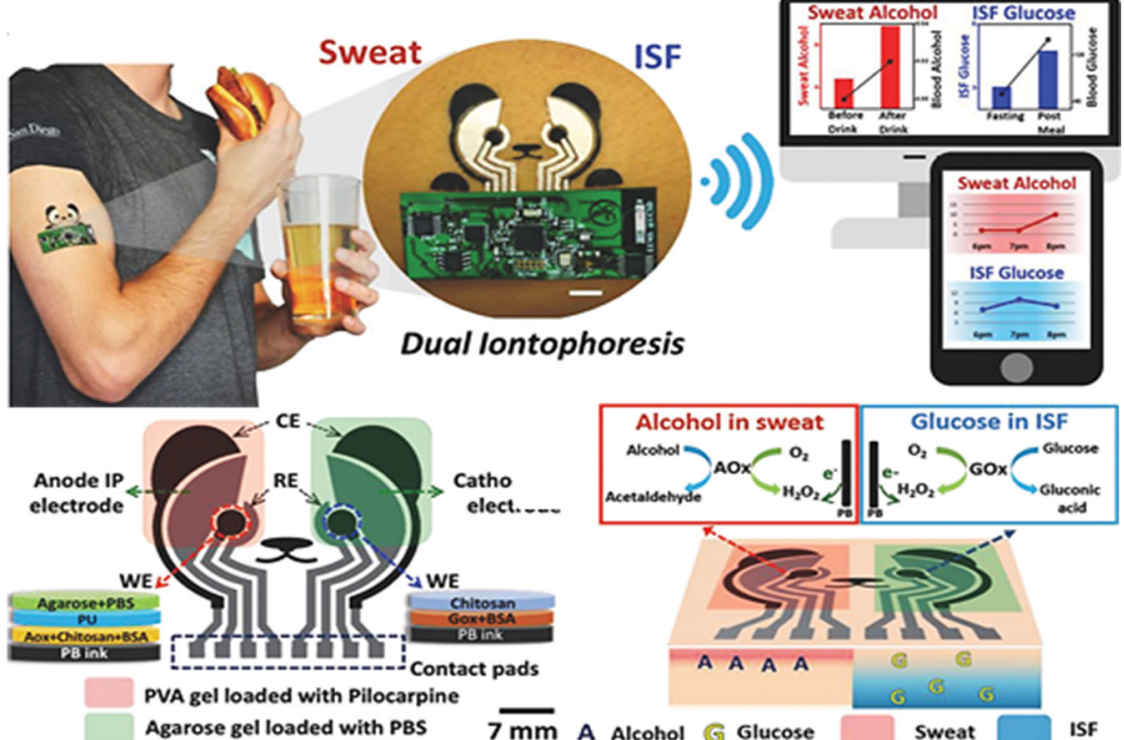

(b)
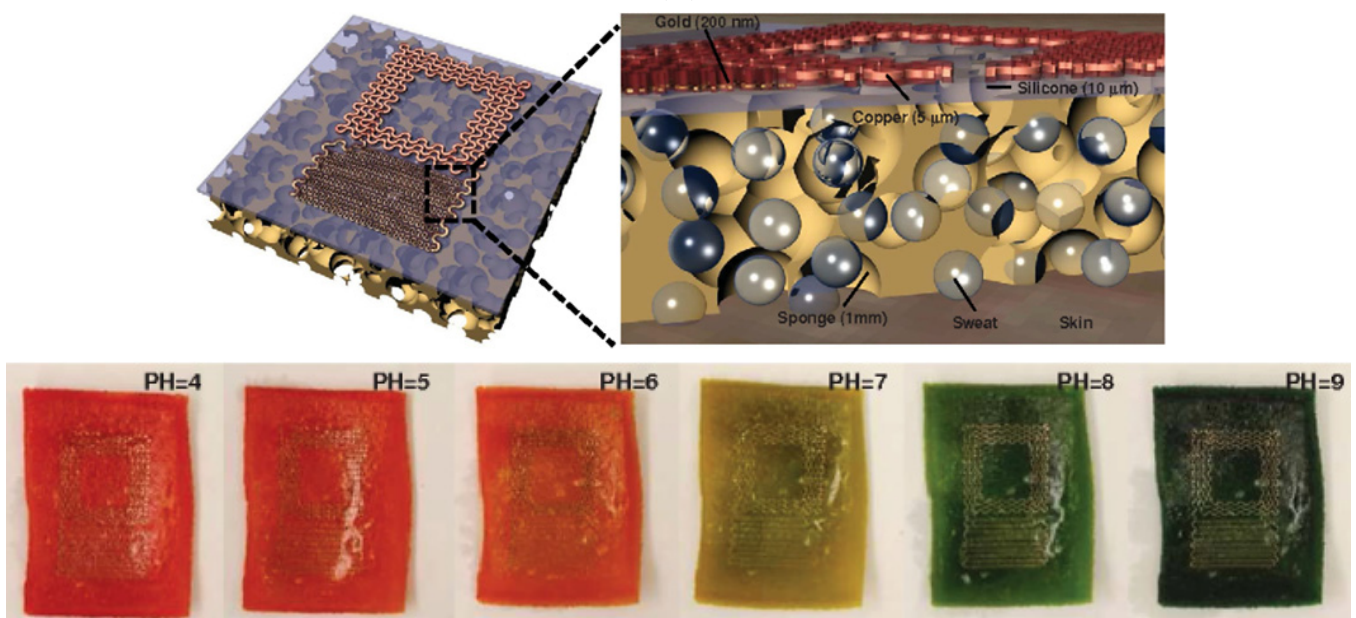

(c)

Figure 18. Cont. 

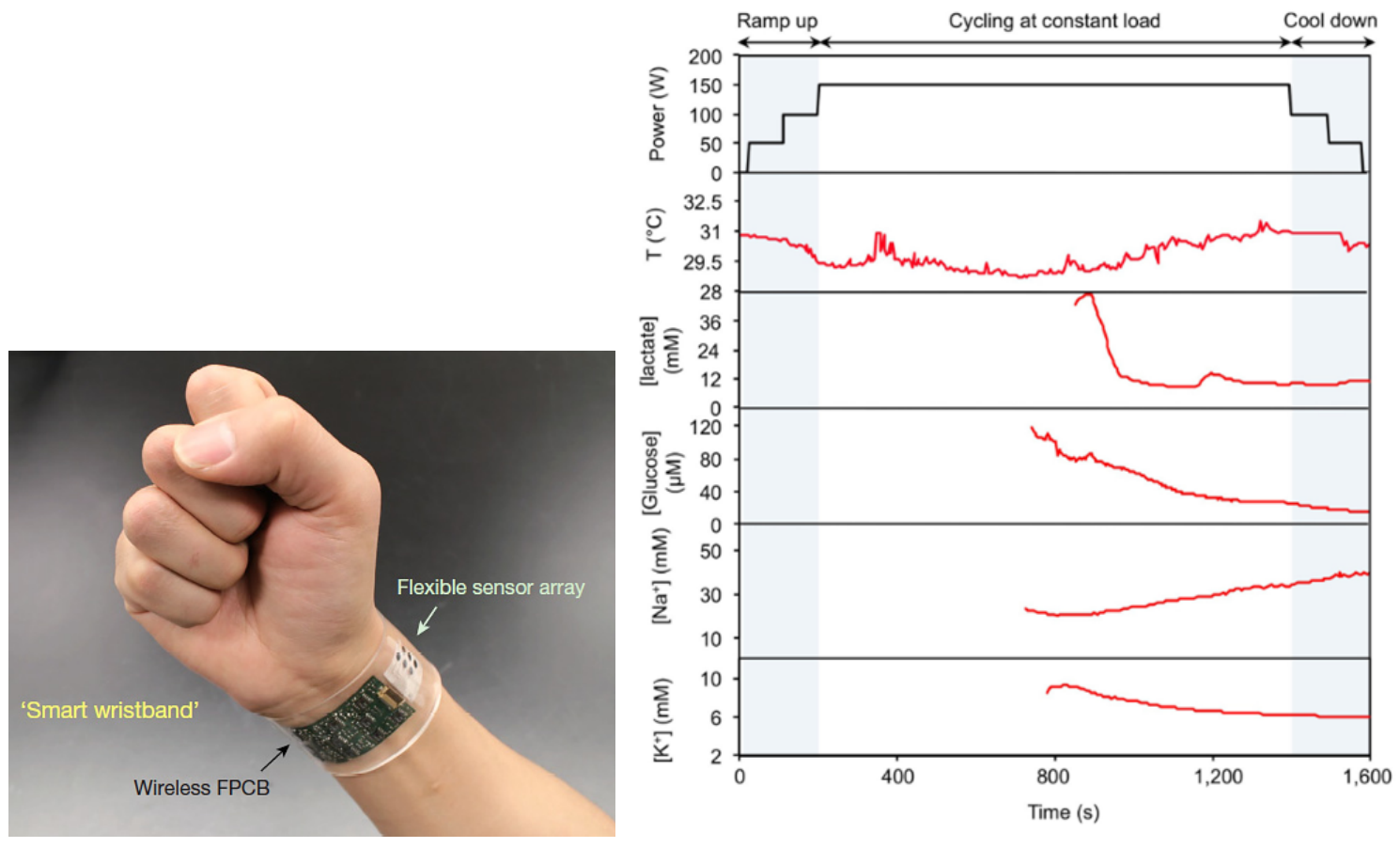

(d)
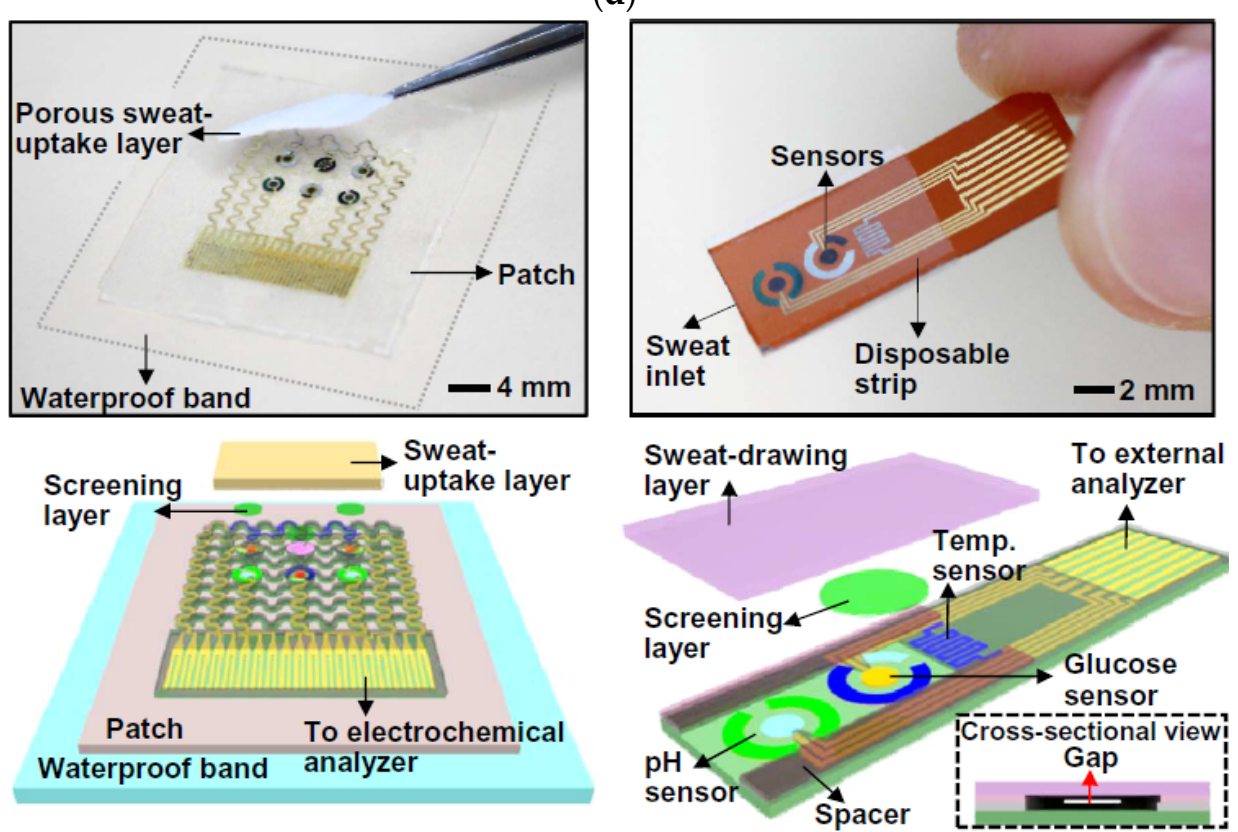

(e)

Figure 18. (a) Functional absorbent materials for sweat sampling in wearable sensors: An ion-selective sensor on a cellular substrate [49] (Reprinted with permission from ref. [49]. Copyright 2021 John Wiley and Sons); (b) wearable biosensors to detect glucose and alcohol on a hydrogel printed tattoo platform [50]; (c) wirelessly connected passive capacitive sensors for $\mathrm{pH}$ sensing [51] (Reprinted with permission from ref. [51]. Copyright 2021 John Wiley and Sons); (d) wearable sweat detection matrix multiplexed with an absorbent thin rayon pad placed between the skin and the sensor matrix for sampling [52] (Reprinted with permission from ref. [52]. Copyright 2021 Springer Nature); (e) wearable sweat monitoring patch (left) and disposable sweat monitoring strip (right) [53]. 


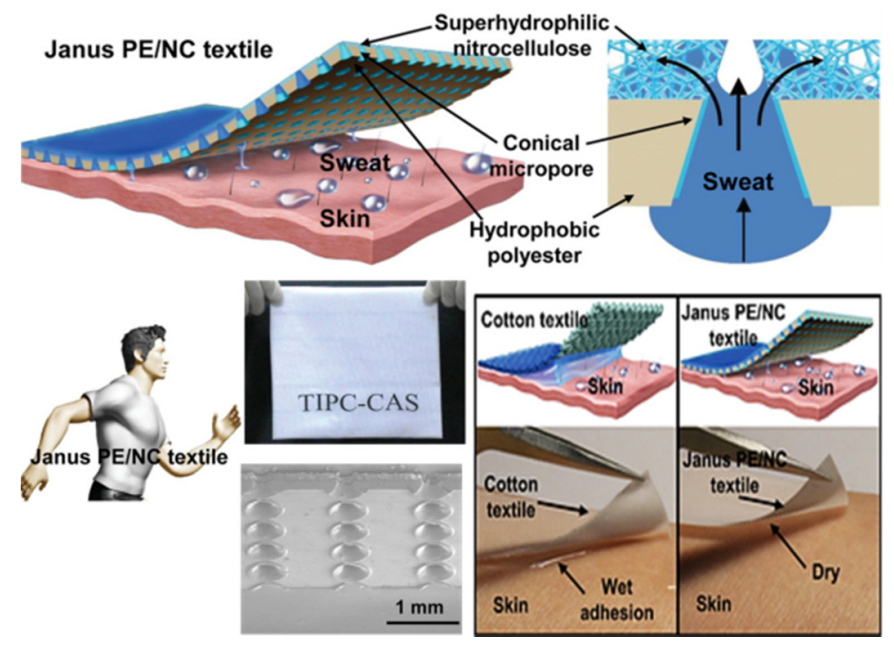

(a)

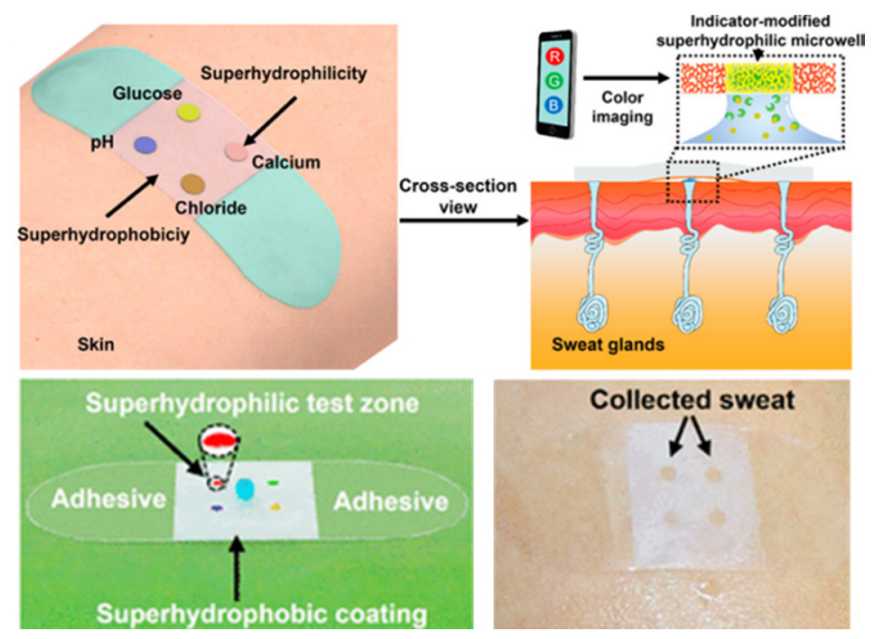

(b)

Figure 19. (a) Super-hydrophobic/super-hydrophilic surface used for sweat sampling in wearable sensors: Janus PE/NC fabric with conical micropores to transport sweat [54] (Reprinted with permission from ref. [54]. Copyright 2021 John Wiley and Sons); (b) a super-wettable band for sweat sampling and multiplex targets analysis [55].

Furthermore, in [56], the authors presented sweat guide channels for sweat collection. Besides, the system was equipped with a conductivity sensor to non-invasively monitor the user's physiological conditions in real-time. Similarly, the wearable sensor platform, called "sweatband", efficiently detects sweat presence, providing real-time analysis of its sodium content [57]. When the subject wore the device, sweat was pumped from the sweat glands through hydraulic pressure during exercise. It was guided into the sensing area using a tube as the pressure increased in the tube, and this was of no significance on the mechanisms of sweating.

Furthermore, in [58], the authors developed a flexible microfluidic patch for sweat detection, constituted by sensors interfaced within a microfluidic channel for real-time electrochemical detection and sweat rate analysis. The sweat penetrated the patch entry regions, guided through a network of microchannels until tanks filled with color-sensitive materials driven by the combination of natural pressure associated with perspiration and capillary effects in the microchannels [59]. Similarly, a device consisting of microchannels for sweat sampling was reported in [60].

In addition, several works are proposed in the literature to non-invasively detect the drugs, hormones, and toxins in the blood. For instance, in [61], a mass-produced, laserengraved microfluidic device was realized for detecting low concentrations of uric acid (UA) and tyrosine (Tyr) in sweat, substances associated with diseases such as gout and metabolic disorders. In addition, in [62], the authors introduced a non-invasive, passive, and simple to use skin surface-based sensing device to determine the blood ethanol content (BAC) and control the transdermal alcohol concentration (TAC). The described system worked in situ, determining the content of ethanol in the blood in real-time by measuring the amperometric signal generated by the ethanol in the sweat, using the pilocarpine iontophoresis test. The detection system included an electrochemical alcohol oxidase/horseradish peroxidase (AOD/HRP) biosensor, a miniaturized potentiostat, and a microprocessor to acquire the analog signal generated by the sweat into the BAC.

Other helpful wearable devices are glove-based ones [63], able to give a rapid estimation of biological and chemical risks, such as explosive [64] and Gunshot Residues (GSR), drugs [65], pesticides [64], and pathogens [66]. These devices are used to perform rapid chemical analysis, allowing law enforcement to carry out on-the-spot qualitative chemical investigations. Practical use of smart glove requires materials, such as flexible electrochemically compatible ones, to resist mechanical deformations and increase functionalities. This kind of device exploits the direct interaction with the tested sample, such as a surface, 
a liquid, as well as a vapor, but to successfully complete the electrochemical analysis, a supporting electrolyte to accomplish the electrochemical circuit is required. Even if ionic liquids could be incorporated in future devices, currently, an aqueous electrolyte limits the on-field application. However, a solid-state ionogel conductive layer, suitably refined and confined using an O-ring, could open new applicative scenarios.

In [67], the authors presented a robotic hand equipped with a chemical sensing skin to taste foodstuffs in liquid and powder form. The system employed an amperometric biosensor printed on the hand surface, differently from the Square Wave Voltammetry (SWV) method used in other systems. Further, in [67], a glove based on the sensing skin is presented, giving the sense of taste to a robot able to analyze different food features, such as the spiciness of paprika and black pepper or the caffeine concentration in beverages.

\section{Overview on IoT Systems for Health Monitoring Applications: Architecture Point of View and Supported Services}

Healthcare systems based on the Internet of Things (IoT) can be applied to a great number of fields, such as care for younger or older patients or the surveillance of chronic disease. In [68], the standard services offered by this kind of system are presented, which potentially need adjustments to become a building block for an application. The services provided by this kind of systems and services are:

- Ambient Assisted Living (AAL), an IoT platform that assists elderly and disabled people thanks to AI techniques for extending and improving their life;

- Internet of m-health things (m-IoT), that consists of mobile computing, medicals sensors, and communication technologies for healthcare services;

- IoT-based Adverse Drug Reaction (ADR), that allows a patient to find a specific solution, according to his allergy profile and electronic health record, for an injury caused by the medicine of assumption;

- Community Healthcare $(\mathrm{CH})$, an IoT-based network that covers an area surrounding a local community to access remote medical advice and health data;

- Children Health Information (CHI) that helps children with behavioral or mental problems by educating and amusing them;

- Wearable Device Access (WDA) that allows remote monitoring of a patient by using non-intrusive sensors equipping mobile computing devices like smartphones;

- Semantic Medical Access (SMA) for sharing medical knowledge and information by using semantics and ontologies;

- Indirect Emergency Healthcare (IEH) that suggests the behavior in dangerous situations like road accidents or adverse weather conditions;

- Embedded Gateway Configuration (EGC), an architectural service that links the Internet and medical devices to the network nodes to which patients are connected;

- Embedded Context Prediction (ECP), a generic framework with a suitable mechanism to create context-aware healthcare applications.

Considering the IoT-based healthcare applications, the authors classify them into two main categories: Single-condition applications related to a specific disease or clusteredcondition applications dealing with a greater diseases' number:

- Glucose level sensing that helps planning meals, activities, and medication by revealing the blood glucose level of patients affected by diabetes;

- Electrocardiogram monitoring to eventually diagnose heart diseases such as arrhythmias and myocardial ischemia by detecting the heart electrical activity;

- Monitoring of blood pressure and body temperature, by using suitable sensors, crucial parameters for healthcare services;

- Oxygen saturation monitoring, useful for technology-driven medical healthcare applications, obtained by combining pulse oximetry with IoT;

- Rehabilitation system for improving the rehabilitation of people with physical impairment or disability; 
- Medication management, that offers several solutions to the non-fulfilment problem in medication, representing a severe menace for public health;

- Wheelchair management, that offers an automatic wheelchair to disabled people;

- Imminent healthcare solutions, that allows numerous portable medical devices to be integrated with IoT networks;

- Healthcare solutions using smartphones, exploiting smartphone functionalities to control electronic devices extending its capabilities in the IoT platform.

The authors proposed IoT healthcare network (IoThNet) platform, constituted by the computing platform and network platform model (Figure 20) [68]; it has a hierarchical structure, allowing caregivers to connect to the application layer's databases using a support layer, obtaining a service platform focused on people's health information.

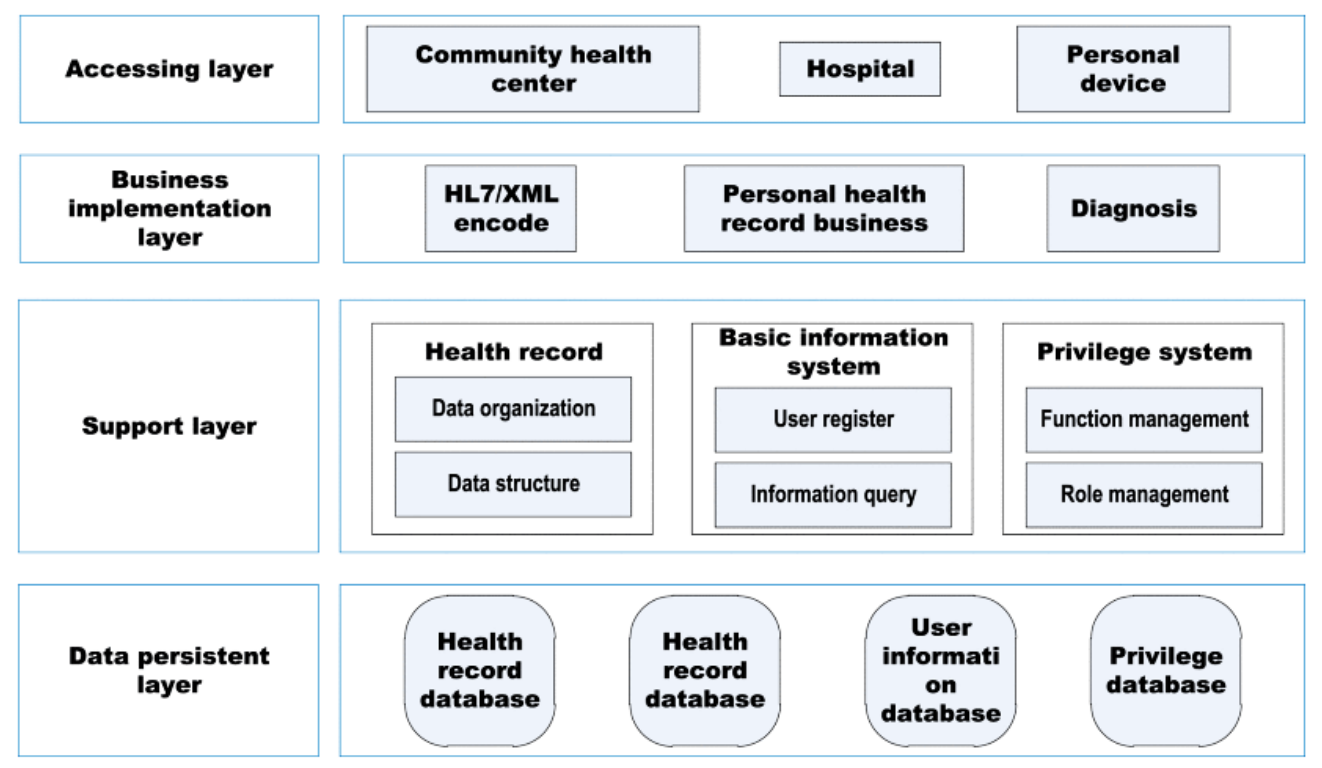

Figure 20. Health information service model's functional framework [68].

Kan et al. presented a Mobile and E-network Smart Health (MESH) system that combines ECG sensing devices for controlling the space-time heart activity of patients [69]. It is Bluetooth-connected to a smartphone, sending the collected data to the MESH cloud via $4 \mathrm{G}$ communication to control the patient's heart conditions (Figure 21).

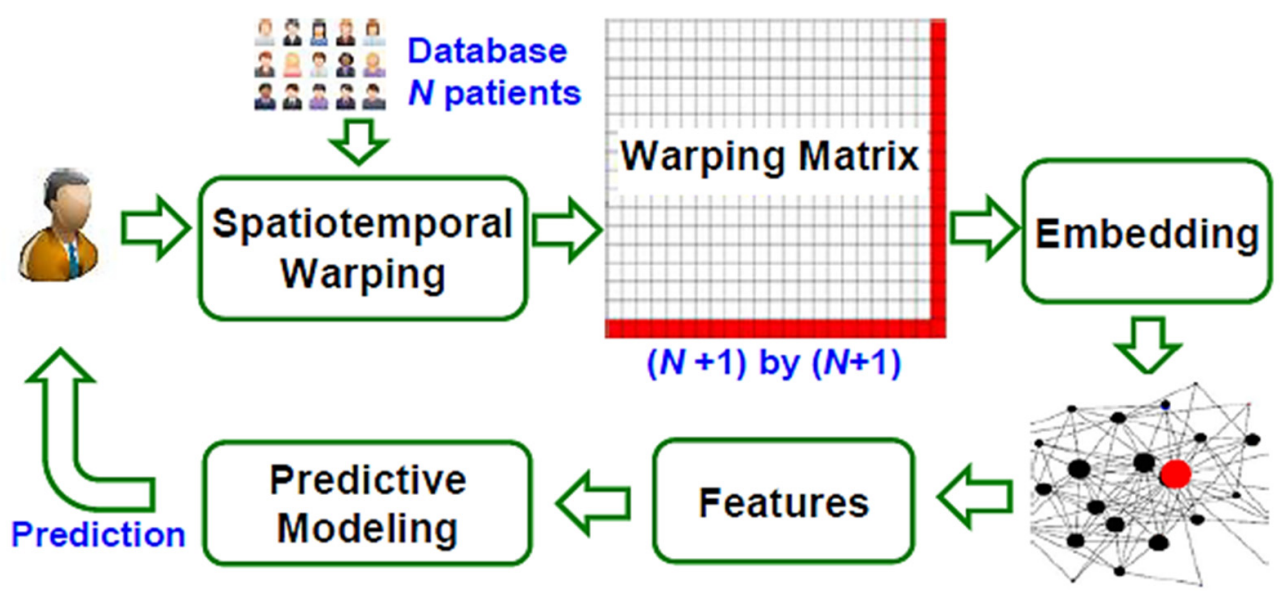

Figure 21. Flow chart of the network-based data analytics in MESH [69] (Reprinted with permission from ref. [69]. Copyright 2021 IEEE). 
This system allows cardiologists to access and control, by authorized mobile devices, the stored data in real-time to identify cardiac diseases early and give timely treatments to life-threatening sickness. If the patient's situation deteriorates, MESH transmits notifications and alarms. To detect and model the heart's electrical activity altered by disease, Vectorcardiogram (VCG) signals were used, constituted by three time-dependent and orthogonal functions $V_{x}=f(t), V_{y}=g(t), V_{z}=h(t)$, providing real-time representation of the cardiac vector. The first function is called "head", representing the actual position of the cardiac vector, whereas the second one is called "body" representing a restricted history of the cardiac vector movements; the third is named "tail", summarizing a full history of vector's movements.

Furthermore, Spanò et al. introduced a system for remote ECG monitoring integrated with an IoT infrastructure, consisted of ECG prototype sensors equipped with the recordlow Energy per Effective Number of Quantized Levels (EEQNL), an architecture enabling the combination of low-cost sensors with other intelligent home systems using the IoT infrastructure (Figure 22) [70].

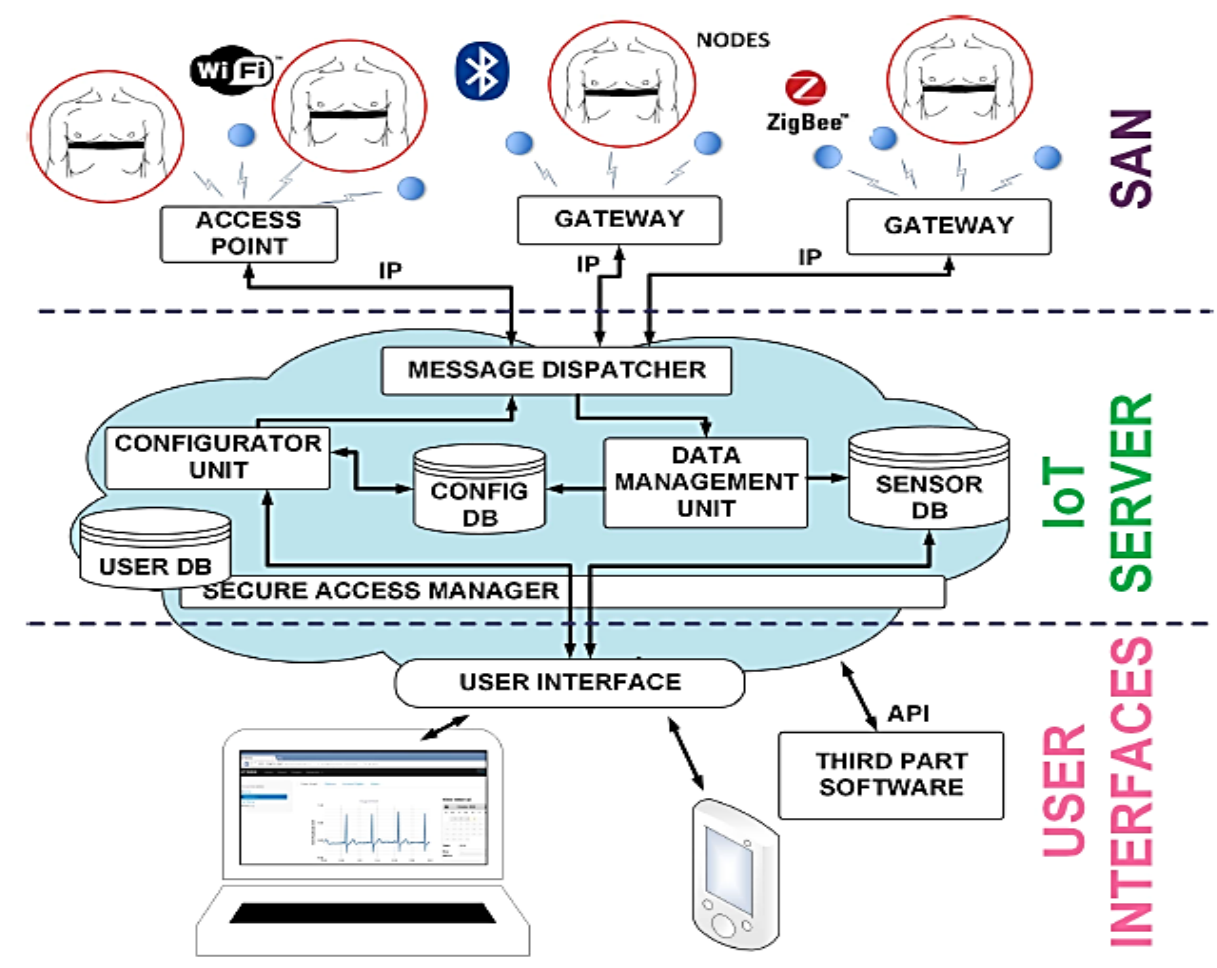

Figure 22. Block diagram of the IoT platform supporting the in-home Smart Grid [70] (Reprinted with permission from ref. [70]. Copyright 2021 IEEE).

Indeed, this ECG monitoring system can be connected to other biomedical ambient monitoring systems at the infrastructure level. At the local deployment level, it can monitor several patients simultaneously using the same wireless infrastructure, reducing the cost to add an eventually new patient. The individual sensor level included proposed ECG sensors, featured by high signal quality and low power consumption. The authors proposed an ECG circuit based on the general-purpose architecture, ensuring high performance and resolution, including a low-power ADC, an analog front-end (AFE), and an adjustable digital filter connected to conductive rubber electrodes installed into a chest belt. The authors estimated device autonomy to about 6 days with compression, 5 without it. The average current consumption of the radio link is about $0.95 \mathrm{~mA}$ with compression and $1.77 \mathrm{~mA}$ without it. 
Moustafa et al. proposed an alternative IoT architecture for remotely controlling patients and managing different medical devices and sensors using Web Real-Time Communication (WebRTC) for supporting the communication between remote users and medical infrastructure [71]. An edge cloud architecture was applied to spare network bandwidth, guaranteeing real-time control of medical devices, using a central communication hub to supply an abstraction layer and aggregate videos and data from different source devices. Moreover, the edge cloud provided storage infrastructure and efficient communication for sensors and devices and acted as a proxy server. Indeed, the IoT gateways worked both as communication and aggregation hubs for videos and data storage, processing, and analytics. The edge cloud also offered push-mode remote monitoring for transmitting warning and alerts messages to the remote user when an anomaly was detected or pullmode remote monitoring remote users to request specific past data and video, guaranteeing an improved privacy policy for stored data.

In [72], the authors presented the Wearable 2.0 healthcare system to enhance performances of future health systems. The Wearable 2.0 framework exploited smart clothes to gather users' physiological data and receive feedback from the cloud platform [72].

The architecture of the Wearable 2.0 healthcare system, depicted in Figure 23, was constituted by a front-end system, a communication system, and a supporting cloud system. The front-end system was composed of various sensors, which acted both like a long-term data source and a user interface. Furthermore, healthcare robots can send alerts and notifications when an illness occurs and collect environmental data so that intelligent clothing provide high mobility. The wireless communication system was fundamental for human-cloud integration, supporting solutions for normal and special users. Normal users employed their smartphone as a personal gateway; in contrast, special users needed access points in areas where frequent activities happen. Finally, the supporting cloud system was the center of different healthcare services and cognitive applications.

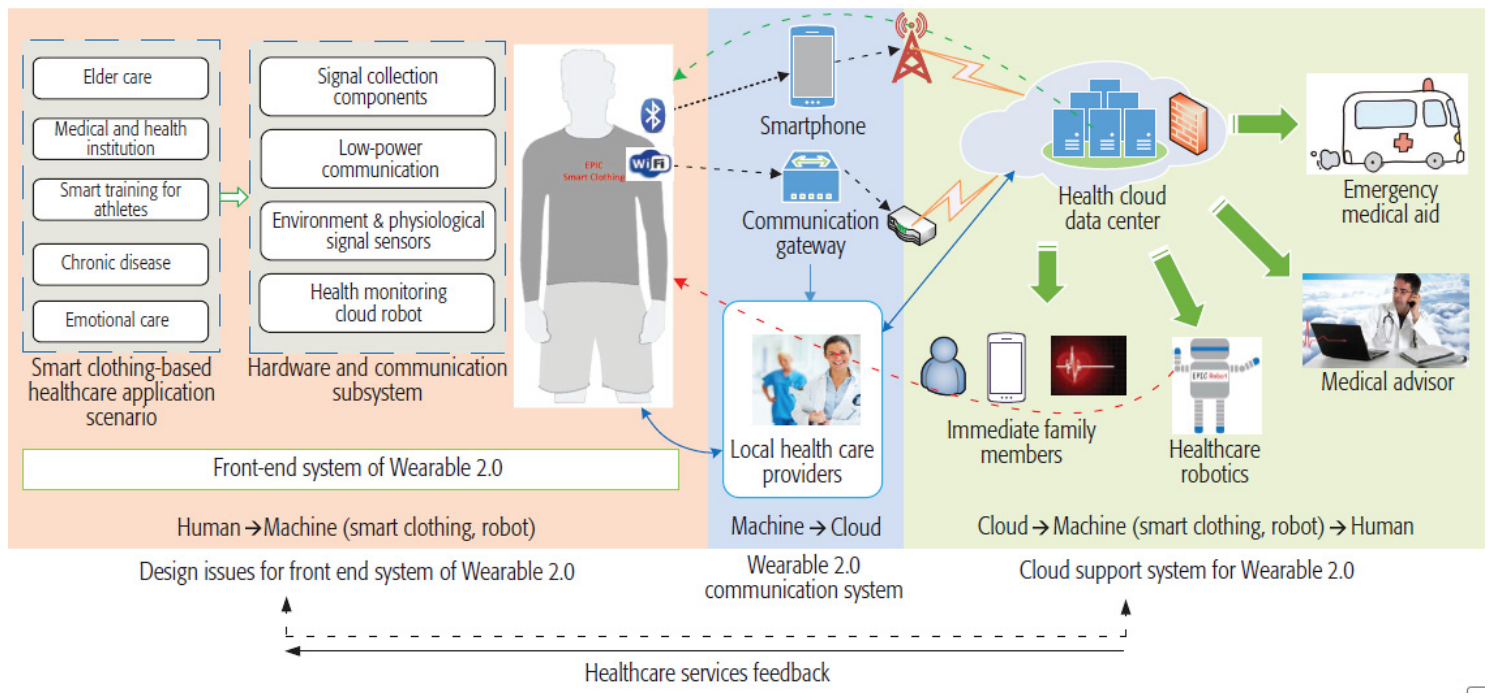

Figure 23. Design for a smart clothing-based healthcare system [72] (Reprinted with permission from ref. [72]. Copyright 2021 IEEE).

Rahmani et al. proposed a geo-distributed intermediary layer based on the concept of fog computing in IoT-based healthcare systems [73]. Their fog-assisted system architecture can face many challenges in ubiquitous healthcare systems, especially in clinical environments, by implementing smart e-Health gateways called UT-GATE [73]. These provided different services at the network edge between smart objects and cloud to satisfy the healthcare sector needs regarding reliability, availability, and robustness. By exploiting the e-Health gateways, an intermediary layer has been implemented to demonstrate fog computing advantages for IoT-based Healthcare systems. The proposed framework was composed of three main components: (1) Medical sensors and actuators network where 
the data gathered from the body and the environment are wirelessly transmitted to the gateway; (2) e-Health gateways network, a layer constituted by a significant number of smart e-Health Gateways connecting the sensor network and the local switch; and (3) a back-end system, a cloud computing platform that provides data analytics.

Farahani et al. stressed the relevance of fog computing as an intermediate layer between devices and the cloud to achieve greater speed, variety, and better latency when analyzing complex data from a patient-centric IoT healthcare system [74]. The proposed system comprised three main layers, viz. IoT eHealth Device Layer, IoT eHealth Fog Layer, and IoT eHealth Cloud Layer. The Device Layer included the medical devices used to monitor users' health conditions, synchronizing collected data with the cloud layer. These devices can be divided into two main categories: (1) Physical sensors that are any medical devices with a wireless connection used to monitor patients' health; (2) Virtual sensors that use mobile applications, software, and eHealth services to collect patient's and environment data. As close as possible to the source devices, the proposed framework employed fog nodes to analyze and compress the acquired data and sent them to the cloud layer. The cloud layer connected the fog nodes and the health devices; also, it integrated the data from different devices, stored them, and made them accessible anytime to patients and doctors. This layer also analyzed the data to generate appropriate alarms and notifications for patients and physicians.

The mobile health (m-health) systems, as discussed by Almotiri et al., would bring many benefits such as rapid diagnosis, home rehabilitation, and remote monitoring [75]. The word m-health identifies the utilization of mobile devices, featured by compactness, low power consumption, IP connectivity, and security, to collect the health data, like blood pressure and blood sugar level, of a patient in real-time. The data were collected using several medical gadgets, such as wearable devices, saving them on network servers connected to the Internet. Thus, the system architecture was a component of the IoT architecture, constituted by multiple layers, namely a data collection layer, a data processing layer, and a data storage layer, as shown in Figure 24.


Data Collection Layer
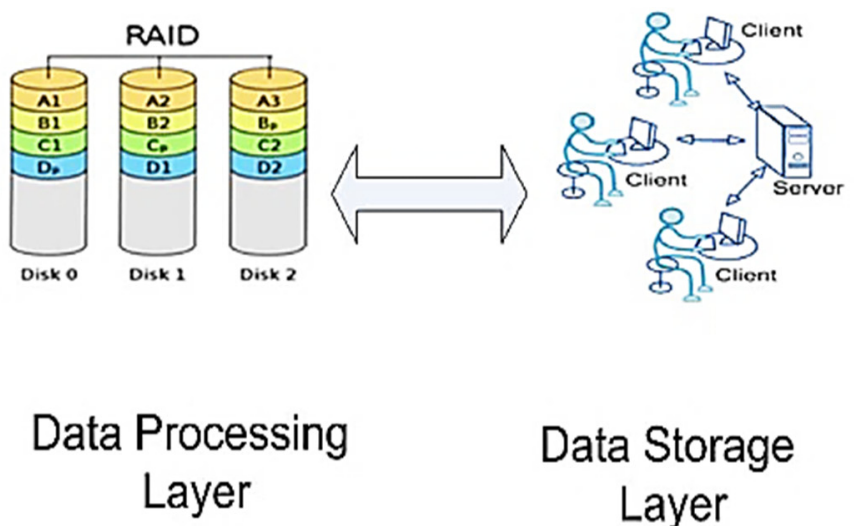

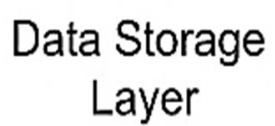

Figure 24. Mobile health (m-health) system architecture, proposed in [75] (Reprinted with permission from ref. [75]. Copyright 2021 IEEE).

Each layer was based on several servers placed in different locations; the data processing layer analyzed the data stored in the storage layer; it comprised IoT servers, allowing the data to be available anywhere. The Health Insurance Portability and Accountability Act (HIPAA) defined the measure to adopt to achieve confidentiality, security, and privacy for the stored health data.

Ambient assisted living would bring many benefits, especially to older people, which is constantly increasing, improving their independent life and ensuring remote monitoring, thus reducing personal assistants. In [76], Rashed et al. presented multi-layer architecture to collect users' health data and environmental information surrounding them. This system 
was constituted by three layers: The perception layer, the network and gateway layer, and the integrated application layer.

The perception layer aggregated the received data and sent them to the next layer; It was divided into two other sub-layers, namely the physical interface and data collection. The physical interface includes different sensors to gather the patient's health data and environmental parameters. The sensors were divided into wearable and unwearable; the wearable sensors are used to acquire biophysical parameters, like HR, body temperature, and motions, crucial to detect an eventual fall for older people. The unwearable sensors acquired the environmental parameters, such as humidity, temperature, and illuminance. The second sub-layer collected and processed the data received from the physical interface and sent it into the next layer.

The network and gateway layer represented a bridge between the perception and the application layer. A Raspberry Pi 3 board (Raspberry Pi Foundation, Cambridge, UK) was used as a gateway in the developed platform, using the Message Queue Telemetry Transport (MQTT) protocol to send the data into the integrated application layer. This has been implemented on a web hosting server. Additionally, the authors implemented a mobile application with a doctor interface to access the patients' profiles, each containing the vital signs collected by the sensors and a patient interface including the recorded history of the collected vital signs.

Besides, IoT-based solutions to detect danger of falling is an ever-current research topic, preventing harmful consequences. Great attention was paid to the fall detection algorithms and frameworks, supported by wearable IoT devices, enabling early detection of dangerous events [77-79]. A common approach is based on inertial sensors, like accelerometers and gyroscope, and data fusion to detect typical movement patterns featuring the fall [80-82]. In fact, falls are featured by greater acceleration values than daily life activities; this approach can induce a high false-positive rate since several activities involve high acceleration values. Improvements can be obtained by analyzing the acceleration data using advanced ML techniques, like neural networks [83-85], K-Nearest Neighbors (KNN) [86], Support Vector Machine (SVM) [87], Naive Bayes [88], etc.

Greene et al. developed an IoT-based fall detection system into a smart home, operating on the local Wi-Fi network [89]. The main components are a central hub (Raspberry Pi board) (Raspberry Pi Foundation, Cambridge, UK), the fall detection device, the Amazon Echo (Amazon, Seattle, WA, USA), a speaker, and a webcam. The fall detection device is based on a Tiva C launchpad (Texas Instruments, Dallas, TX, USA) with a Wi-Fi expansion board interfaced with the ADXL345 accelerometer (Adafruit Industries, New York, NY, USA). The detection algorithm exploits threshold-based interrupts, generated if the acceleration is over-threshold for a set time. Pierleoni et al. proposed a fall detection device with an MPU-6050 three-axis accelerometer and gyroscope (SparkFun Electronics, Boulder, CO, USA), an HMC5883L magnetometer (Honeywell, Plymouth, MA, USA), and an MS5611-01BA barometer (TE Connectivity, Schaffhausen, Switzerland), providing information on the user's altitude and posture [90]. The ATmega328 microcontroller (Atmel, San Jose, CA, USA) collects data from sensors, executes the fall detection algorithm, based on opportune thresholds, and wirelessly sends the alarms using a Bluetooth HC-05 module (manufactured by Nisang Robotics Technology, Mumbai, India).

The development of non-intrusive systems for monitoring Activities of Daily Living (ADL), such as medication intake, is crucial to support clinical professionals and enhance healthcare services. Liu et al. developed a system, based on wearable sensors, for recognizing housekeeping tasks and classifying the corresponding activity level, essential for several clinical assessment tools [91]. The system architecture is depicted in Figure 25; the movement data were collected using a wearable device, called OPAL, equipped with three-axis gyroscopes and accelerometers and wirelessly sent to a laptop for pre-processing. The moving average technique recognizes activity and removes noise, whereas the sliding window method efficiently divides time data series into ten segments. One segment is used to test the classifier, the remaining nine for training the ML model. A feature vector is built 
during the training phase by collecting physical features by the pre-processed segments. The Principal Component Analysis extracts significant features (i.e., root mean square and deviation) to build a classifier for recognizing and classifying the housekeeping activity (accuracy of $90.67 \%$ and $94.35 \%$, respectively).

\section{Data Acquisition}

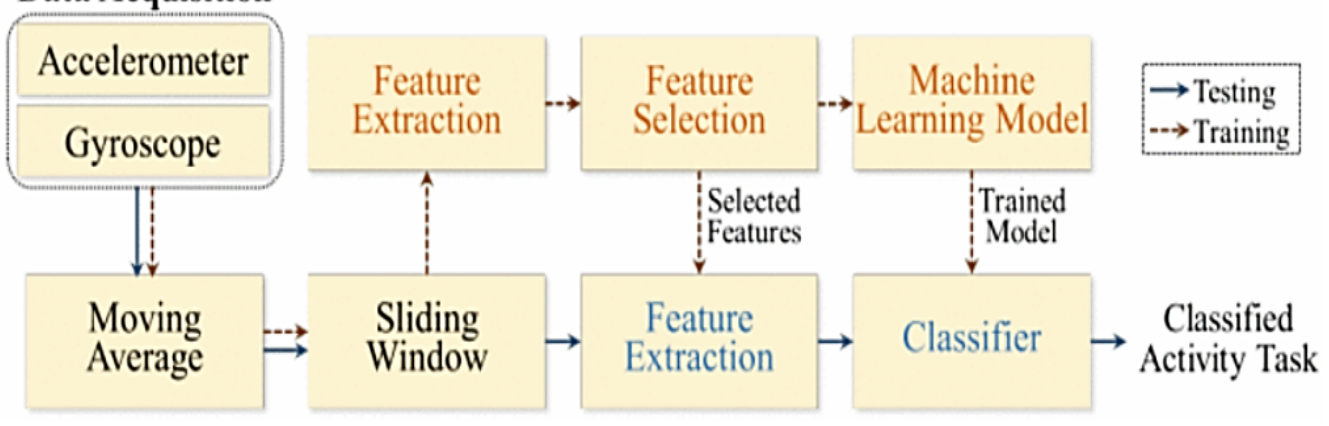

Figure 25. Architecture of the proposed housekeeping task monitoring system [91] (Reprinted with permission from ref. [91]. Copyright 2021 IEEE).

In conclusion, Table 1 summarizes the scientific works previously analyzed from the point of view of the availability of a fog/edge computing layer, the storage modality, and the typology of provided feedback.

Table 1. Summary of the scientific works previously discussed from the point of view of the availability of a fog/edge layer, the storage modality, and the typology of provided feedback.

\begin{tabular}{|c|c|c|c|}
\hline Work & Edge/Fog Computing & Storage Modality & Feedback Typology \\
\hline C. Kan et al. [69] & $\begin{array}{l}\text { Smartphone provided pre-processing } \\
\text { capability and network connectivity }\end{array}$ & Central DB & Prediction about heart diseases \\
\hline E. Spanò et al. [70] & $\begin{array}{l}\text { Gateway collected and processed data } \\
\text { from wearables devices }\end{array}$ & Central DB & $\begin{array}{l}\text { Information about heart } \\
\text { dysfunctions }\end{array}$ \\
\hline H. Moustafa et al. [71] & $\begin{array}{l}\text { Edge Cloud of Gateway performed data } \\
\text { aggregation from wearable devices }\end{array}$ & Local and Central DB & $\begin{array}{l}\text { Information about health and } \\
\text { wellness of user's status }\end{array}$ \\
\hline M. Chen et al. [72] & Local Health providers & Central DB & $\begin{array}{l}\text { Emergency medical aid } \\
\quad \text { Medical advisor } \\
\text { Warning to family members }\end{array}$ \\
\hline A.M. Rahmani et al. [73] & $\begin{array}{c}\text { Gateways carried out data processing, } \\
\text { local notification, protocol conversion, } \\
\text { data filtering and mining }\end{array}$ & Local and Central DB & $\begin{array}{l}\text { Emergency medical aid } \\
\quad \text { Medical advisor } \\
\text { Warning to family members }\end{array}$ \\
\hline B. Farahani et al. [74] & $\begin{array}{l}\text { Fog nodes provided network connectivity, } \\
\text { computational power and storage }\end{array}$ & Local and Central DB & $\begin{array}{c}\text { Emergency medical aid } \\
\quad \text { Medical advisor } \\
\text { Warning to family members }\end{array}$ \\
\hline A. Rashed et al. [76] & $\begin{array}{l}\text { Gateways carried out data processing, } \\
\text { local notification, protocol conversion }\end{array}$ & Local and Central DB & $\begin{array}{c}\text { Medical advisor } \\
\text { Information about health and } \\
\text { wellness of user's status }\end{array}$ \\
\hline S. Green et al. [89] & - & Local and Central DB & $\begin{array}{l}\text { Fall Detection, Warning messages } \\
\text { to family and caregivers }\end{array}$ \\
\hline
\end{tabular}

\section{Conclusions}

Information and communication technologies offer seamless opportunities to connect people and objects, simplifying and enriching our lives. Due to the widespread use of IoT platforms promoting advanced sensing systems and intelligent inferring methodologies, health systems are undergoing a true technical revolution, fundamentally transforming the way medical services are delivered.

This review paper analyzes the recent advances in sensing systems and technologies suitable for wearable and portable devices to monitor the biophysical and psychophysical 
parameters, offering useful insights for the next generation of smart wearable devices. Subsequently, an overview of IoT platforms for health monitoring is presented, focusing on architectural aspects and the different typologies of offered services; such platforms constitute the basis for the future health care systems, allowing remote, non-invasive, and reliable detection of the patients' conditions. In particular, we focused on IoT healthcare platforms supported by wearable devices for the capillary, accurate, and real-time detection of patient conditions.

From the work that has been carried out, we can state that the future trend for the development of IoT-based platforms will see a paradigm shift passing from centralized architectures to distributed frameworks, based on the concepts of fog and edge computing, allowing to allocate computational, storage, and control resources to a multi-layer architecture, thus enabling great flexibility and computational power, high speed, and low latency [92,93]. Furthermore, this new approach will provide more services closer to the user, hardly supported by a centralized cloud architecture, opening new scenarios in different application fields such as healthcare, smart cities, home automation, etc.

Author Contributions: Conceptualization, R.D.F. and P.V.; investigation, R.D.F. and P.V.; resources, R.D.F.; data curation, P.V.; writing-original draft preparation, R.D.F. and P.V.; writing-review and editing, M.D.V. and P.V.; visualization, P.V. and R.D.F.; supervision, M.D.V. All authors have read and agreed to the published version of the manuscript.

Funding: This research received no external funding.

Informed Consent Statement: Informed consent was obtained from all subjects involved in the study.

Data Availability Statement: Data of our study are available upon request.

Conflicts of Interest: The authors declare no conflict of interest.

\section{References}

1. Chan, M.; Estève, D.; Fourniols, J.-Y.; Escriba, C.; Campo, E. Smart Wearable Systems: Current Status and Future Challenges. Artif. Intell. Med. 2012, 56, 137-156. [CrossRef]

2. Frischer, R.; Krejcar, O.; Maresova, P.; Fadeyi, O.; Selamat, A.; Kuca, K.; Tomsone, S.; Teixeira, J.P.; Madureira, J.; Melero, F.J. Commercial ICT Smart Solutions for the Elderly: State of the Art and Future Challenges in the Smart Furniture Sector. Electronics 2020, 9, 149. [CrossRef]

3. Lobaccaro, G.; Carlucci, S.; Löfström, E. A Review of Systems and Technologies for Smart Homes and Smart Grids. Energies 2016, 9, 348. [CrossRef]

4. de Fazio, R.; Sponziello, A.; Cafagna, D.; Velazquez, R.; Visconti, P. An Overview on Technologies and Devices against Covid-19 Pandemic Diffusion: Virus Detection and Monitoring Solutions. Int. J. Smart Sens. Intell. Syst. 2021, 14, 1-28. [CrossRef]

5. Latifi, R. Current Principles and Practices of Telemedicine and e-Health, 1st ed.; Ios Press, Inc.: Amsterdam, The Netherlands; Washington, DC, USA, 2008; ISBN 978-1-58603-806-9.

6. Wen, H.J.; Tan, J. The Evolving Face of Telemedicine E-Health: Opening Doors and Closing Gaps in e-Health Services Opportunities Challenges. In Proceedings of the 36th Annual Hawaii International Conference on System Sciences, Big Island, HI, USA, 6-9 January 2003; pp. 1-12.

7. Surantha, N.; Atmaja, P.; David; Wicaksono, M. A Review of Wearable Internet-of-Things Device for Healthcare. Procedia Comput. Sci. 2021, 179, 936-943. [CrossRef]

8. Nasajpour, M.; Pouriyeh, S.; Parizi, R.M.; Dorodchi, M.; Valero, M.; Arabnia, H.R. Internet of Things for Current COVID-19 and Future Pandemics: An Exploratory Study. J. Healthc. Inf. Res. 2020, 4, 325-364. [CrossRef] [PubMed]

9. Singh, R.P.; Javaid, M.; Haleem, A.; Suman, R. Internet of Things (IoT) Applications to Fight against COVID-19 Pandemic. Diabetes Metab. Syndr. 2020, 14, 521-524. [CrossRef] [PubMed]

10. Dong, Y.; Yao, Y.-D. IoT Platform for COVID-19 Prevention and Control: A Survey. IEEE Access 2021, 9, 49929-49941. [CrossRef]

11. Kadhim, K.T.; Alsahlany, A.M.; Wadi, S.M.; Kadhum, H.T. An Overview of Patient's Health Status Monitoring System Based on Internet of Things (IoT). Wirel. Pers. Commun. 2020, 114, 2235-2262. [CrossRef]

12. Ennafiri, M.; Mazri, T. Internet of Things for Smart Healthcare: A Review on a Potential IOT Based System and Technologies to Control COVID-19 Pandemic. Innov. Smart Cities Appl. 4 2020, 183, 1256-1269. [CrossRef]

13. Zhang, L.; Yang, L.; Wang, Z.; Yan, D. Sports Wearable Device Design and Health Data Monitoring Based on Wireless Internet of Things. Microprocess. Microsyst. 2020, 103423, 1-7. [CrossRef]

14. Putri, A.; Ali, M.; Abdulmajid, M.S.; Hidayat, S.S. Wearable Sensor and Internet of Things Technology for Better Medical Science: A Review. Int. J. Emerg. Technol. Learn. (IJET) 2019, 7, 1-4. [CrossRef] 
15. Burmaoglu, S.; Trajkovik, V.; Tutukalo, T.L.; Yalcin, H.; Caulfield, B. Chapter 14-Evolution Map of Wearable Technology Patents for Healthcare Field. In Wearable Technology in Medicine and Health Care; Tong, R.K.-Y., Ed.; Academic Press: Cambridge, MA, USA, 2018; pp. 275-290, ISBN 978-0-12-811810-8.

16. Honarvar, M.G.; Latifi, M. Overview of Wearable Electronics and Smart Textiles. J. Text. Inst. 2017, 108, 631-652. [CrossRef]

17. Stavropoulos, T.G.; Papastergiou, A.; Mpaltadoros, L.; Nikolopoulos, S.; Kompatsiaris, I. IoT Wearable Sensors and Devices in Elderly Care: A Literature Review. Sensors 2020, 20, 2826. [CrossRef]

18. de Fazio, R.; Cafagna, D.; Marcuccio, G.; Minerba, A.; Visconti, P. A Multi-Source Harvesting System Applied to Sensor-Based Smart Garments for Monitoring Workers' Bio-Physical Parameters in Harsh Environments. Energies 2020, 13, 2161. [CrossRef]

19. Jayathilaka, W.A.D.M.; Qi, K.; Qin, Y.; Chinnappan, A.; Serrano-García, W.; Baskar, C.; Wang, H.; He, J.; Cui, S.; Thomas, S.W.; et al. Significance of Nanomaterials in Wearables: A Review on Wearable Actuators and Sensors. Adv. Mater. 2019, 31, 1-21. [CrossRef]

20. Wang, C.; Xia, K.; Wang, H.; Liang, X.; Yin, Z.; Zhang, Y. Advanced Carbon for Flexible and Wearable Electronics. Adv. Mater. 2019, 31, 1-37. [CrossRef] [PubMed]

21. Lim, H.-R.; Kim, H.S.; Qazi, R.; Kwon, Y.-T.; Jeong, J.-W.; Yeo, W.-H. Advanced Soft Materials, Sensor Integrations, and Applications of Wearable Flexible Hybrid Electronics in Healthcare, Energy, and Environment. Adv. Mater. 2020, $32,1-43$. [CrossRef]

22. Nagai, Y.; Jones, C.I.; Sen, A. Galvanic Skin Response (GSR)/Electrodermal/Skin Conductance Biofeedback on Epilepsy: A Systematic Review and Meta-Analysis. Front. Neurol. 2019, 10, 1-8. [CrossRef]

23. Anwar, Y.; Khan, A.A. Assistive Technologies for Bipolar Disorder: A Survey. Int. J. Adv. Comput. Sci. Appl. 2019, 10, 490-499. [CrossRef]

24. Malathi, D.; Jayaseeli, J.D.; Madhuri, S.; Senthilkumar, K. Electrodermal Activity Based Wearable Device for Drowsy Drivers. J. Phys. Conf. Ser. 2018, 1000, 1-11. [CrossRef]

25. Chung, M.; Fortunato, G.; Radacsi, N. Wearable Flexible Sweat Sensors for Healthcare Monitoring: A Review. J. R. Soc. Interface 2019, 16, 1-15. [CrossRef]

26. Paul, A.; Pinjari, H.; Hong, W.-H.; Seo, H.C.; Rho, S. Fog Computing-Based IoT for Health Monitoring System. J. Sens. 2018, 2018, 1-8. [CrossRef]

27. Aldahiri, A.; Alrashed, B.; Hussain, W. Trends in Using IoT with Machine Learning in Health Prediction System. Forecasting 2021, 3, 181-206. [CrossRef]

28. Vallabh, P.; Malekian, R.; Ye, N.; Bogatinoska, D.C. Fall Detection Using Machine Learning Algorithms. In Proceedings of the 2016 24th International Conference on Software, Telecommunications and Computer Networks (SoftCOM), Split, Croatia, 22-24 September 2016; pp. 1-9.

29. Hong, L.; Deye, Y.; Andrew, B.; Naresh, V.; Jing, W.; Jia, W.S.; Pu, Z.P.; Genyue, F.; Kang, L.; Feng, Z.-P. Smartphone-Based Blood Pressure Measurement Using Transdermal Optical Imaging Technology. Circ. Cardiovasc. Imaging 2019, 12, 1-30. [CrossRef]

30. Chandrasekhar, A.; Kim, C.-S.; Naji, M.; Natarajan, K.; Hahn, J.-O.; Mukkamala, R. Smartphone-Based Blood Pressure Monitoring via the Oscillometric Finger-Pressing Method. Sci. Transl. Med. 2018, 10, 1-10. [CrossRef] [PubMed]

31. Chen, S.; Wu, N.; Lin, S.; Duan, J.; Xu, Z.; Pan, Y.; Zhang, H.; Xu, Z.; Huang, L.; Hu, B.; et al. Hierarchical Elastomer Tuned Self-Powered Pressure Sensor for Wearable Multifunctional Cardiovascular Electronics. Nano Energy 2020, 70, 1-9. [CrossRef]

32. Zambrana-Vinaroz, D.; Vicente-Samper, J.M.; Juan, C.G.; Esteve-Sala, V.; Sabater-Navarro, J.M. Non-Invasive Device for Blood Pressure Wave Acquisition by Means of Mechanical Transducer. Sensors 2019, 19, 4311. [CrossRef] [PubMed]

33. Haxha, S.; Jhoja, J. Optical Based Noninvasive Glucose Monitoring Sensor Prototype. IEEE Photonics J. 2016, 8, 1-11. [CrossRef]

34. Sim, J.Y.; Ahn, C.-G.; Jeong, E.-J.; Kim, B.K. In Vivo Microscopic Photoacoustic Spectroscopy for Non-Invasive Glucose Monitoring Invulnerable to Skin Secretion Products; Scientific Reports. Nature 2018, 8, 1-11. [CrossRef]

35. Rachim, V.P.; Chung, W.-Y. Wearable-Band Type Visible-near Infrared Optical Biosensor for Non-Invasive Blood Glucose Monitoring. Sens. Actuators B Chem. 2019, 286, 173-180. [CrossRef]

36. Villarejo, M.V.; Zapirain, B.G.; Zorrilla, A.M. A Stress Sensor Based on Galvanic Skin Response (GSR) Controlled by ZigBee. Sensors 2012, 12, 6075-6101. [CrossRef]

37. Raj, J.V.; Sarath, T.V. An IoT Based Real-Time Stress Detection System for Fire-Fighters. In Proceedings of the 2019 International Conference on Intelligent Computing and Control Systems (ICCS), Madurai, India, 15-17 May 2019; pp. 354-360.

38. Bayo-Monton, J.-L.; Martinez-Millana, A.; Han, W.; Fernandez-Llatas, C.; Sun, Y.; Traver, V. Wearable Sensors Integrated with Internet of Things for Advancing EHealth Care. Sensors 2018, 18, 1851. [CrossRef]

39. Freivalds, A.; Lyu, S. Body Balance Estimation in Standing and Walking Conditions Using Inertial Measurement Units and Galvanic Skin Response. In Proceedings of the 20th Congress of the International Ergonomics Association (IEA 2018); Bagnara, S., Tartaglia, R., Albolino, S., Alexander, T., Fujita, Y., Eds.; Springer International Publishing: Cham, Switzerland, 2019 ; pp. 9-12.

40. Du, Y. Fall Detection with Galvanic Skin Response Sensor. Thesis, Nanyang Technology University, Singapore, 2019. Available online: https:/ / dr.ntu.edu.sg/handle/10356/78931 (accessed on 21 June 2021).

41. Kikhia, B.; Stavropoulos, T.G.; Andreadis, S.; Karvonen, N.; Kompatsiaris, I.; Sävenstedt, S.; Pijl, M.; Melander, C. Utilizing a Wristband Sensor to Measure the Stress Level for People with Dementia. Sensors 2016, 16, 1989. [CrossRef] [PubMed] 
42. Acerbi, G.; Rovini, E.; Betti, S.; Tirri, A.; Rónai, J.F.; Sirianni, A.; Agrimi, J.; Eusebi, L.; Cavallo, F. A Wearable System for Stress Detection Through Physiological Data Analysis. In Proceedings of the Ambient Assisted Living; Casiddu, N., Porfirione, C., Monteriù, A., Cavallo, F., Eds.; Springer International Publishing: Cham, Switzerland, 2017; pp. 31-50.

43. Navea, R.F.; Buenvenida, P.J.; Cruz, C.D. Stress Detection Using Galvanic Skin Response: An Android Application. J. Phys. Conf. Ser. 2019, 1372, 1-7. [CrossRef]

44. Hernando Gallego, F.; Luengo, D.; Artés Rodríguez, A. Feature Extraction of Galvanic Skin Responses by Non-Negative Sparse Deconvolution. IEEE J. Biomed. Health Inform. 2017, 22, 1385-1394. [CrossRef] [PubMed]

45. Lestari, G.R.; Abuzairi, T. Design of Portable Galvanic Skin Response Sensor for Pain Sensor. In Proceedings of the 2020 International Conference on Smart Technology and Applications (ICoSTA), Surabaya, Indonesia, 20 February 2020; pp. 1-5.

46. Kyriakou, K.; Resch, B.; Sagl, G.; Petutschnig, A.; Werner, C.; Niederseer, D.; Liedlgruber, M.; Wilhelm, F.H.; Osborne, T.; Pykett, J. Detecting Moments of Stress from Measurements of Wearable Physiological Sensors. Sensors 2019, 19, 3805. [CrossRef]

47. Mahmud, M.S.; Wang, H.; Fang, H. SensoRing: An Integrated Wearable System for Continuous Measurement of Physiological Biomarkers. In Proceedings of the 2018 IEEE International Conference on Communications (ICC), Kansas City, MO, USA, 20-24 May 2018; pp. 1-7.

48. Schiavoni, R.; Monti, G.; Piuzzi, E.; Tarricone, L.; Tedesco, A.; De Benedetto, E.; Cataldo, A. Feasibility of a Wearable Reflectometric System for Sensing Skin Hydration. Sensors 2020, 20, 2833. [CrossRef]

49. Lee, Y.K.; Jang, K.-I.; Ma, Y.; Koh, A.; Chen, H.; Jung, H.N.; Kim, Y.; Kwak, J.W.; Wang, L.; Xue, Y.; et al. Chemical Sensing Systems That Utilize Soft Electronics on Thin Elastomeric Substrates with Open Cellular Designs. Adv. Funct. Mater. 2017, 27, 1-8. [CrossRef]

50. Kim, J.; Sempionatto, J.R.; Imani, S.; Hartel, M.C.; Barfidokht, A.; Tang, G.; Campbell, A.S.; Mercier, P.P.; Wang, J. Simultaneous Monitoring of Sweat and Interstitial Fluid Using a Single Wearable Biosensor Platform. Adv. Sci. 2018, 5, 1-11. [CrossRef] [PubMed]

51. Huang, X.; Huang, X.; Liu, Y.; Chen, K.; Shin, W.-J.; Lu, C.-J.; Kong, G.-W.; Patnaik, D.; Lee, S.-H.; Cortes, J.F.; et al. Stretchable, Wireless Sensors and Functional Substrates for Epidermal Characterization of Sweat. Nano Micro Small 2014, 10, 3083-3090. [CrossRef] [PubMed]

52. Gao, W.; Emaminejad, S.; Nyein, H.Y.Y.; Challa, S.; Chen, K.; Peck, A.; Fahad, H.M.; Ota, H.; Shiraki, H.; Kiriya, D.; et al. Fully Integrated Wearable Sensor Arrays for Multiplexed in Situ Perspiration Analysis. Nature 2016, 529, 509-514. [CrossRef] [PubMed]

53. Lee, H.; Song, C.; Hong, Y.S.; Kim, M.S.; Cho, H.R.; Kang, T.; Shin, K.; Choi, S.H.; Hyeon, T.; Kim, D.-H. Wearable/Disposable Sweat-Based Glucose Monitoring Device with Multistage Transdermal Drug Delivery Module. Sci. Adv. 2017, 3, 1-9. [CrossRef]

54. Dai, B.; Li, K.; Shi, L.; Wan, X.; Liu, X.; Zhang, F.; Jiang, L.; Wang, S. Bioinspired Janus Textile with Conical Micropores for Human Body Moisture and Thermal Management. Adv. Mater. 2019, 13, 1-7. [CrossRef]

55. He, X.; Xu, T.; Gu, Z.; Gao, W.; Xu, L.-P.; Pan, T.; Zhang, X. Flexible and Superwettable Bands as a Platform toward Sweat Sampling and Sensing. Anal. Chem. 2019, 91, 4296-4300. [CrossRef]

56. Liu, G.; Ho, C.; Slappey, N.; Zhou, Z.; Snelgrove, S.E.; Brown, M.; Grabinski, A.; Guo, X.; Chen, Y.; Miller, K.; et al. A Wearable Conductivity Sensor for Wireless Real-Time Sweat Monitoring. Sens. Actuators B Chem. 2016, 227, 35-42. [CrossRef]

57. Wang, S.; Wu, Y.; Gu, Y.; Li, T.; Luo, H.; Li, L.-H.; Bai, Y.; Li, L.; Liu, L.; Cao, Y.; et al. Wearable Sweatband Sensor Platform Based on Gold Nanodendrite Array as Efficient Solid Contact of Ion-Selective Electrode. Anal. Chem. 2017, 89, 10224-10231. [CrossRef]

58. Nyein, H.Y.Y.; Tai, L.-C.; Ngo, Q.P.; Chao, M.; Zhang, G.B.; Gao, W.; Bariya, M.; Bullock, J.; Kim, H.; Fahad, H.M.; et al. A Wearable Microfluidic Sensing Patch for Dynamic Sweat Secretion Analysis. ACS Sens. 2018, 3, 944-952. [CrossRef]

59. Koh, A.; Kang, D.; Xue, Y.; Lee, S.; Pielak, R.M.; Kim, J.; Hwang, T.; Min, S.; Banks, A.; Bastien, P.; et al. A Soft, Wearable Microfluidic Device for the Capture, Storage, and Colorimetric Sensing of Sweat. Sci. Transl. Med. 2016, 8, 1-24. [CrossRef]

60. Reeder, J.T.; Choi, J.; Xue, Y.; Gutruf, P.; Hanson, J.; Liu, M.; Ray, T.; Bandodkar, A.J.; Avila, R.; Xia, W.; et al. Waterproof, Electronics-Enabled, Epidermal Microfluidic Devices for Sweat Collection, Biomarker Analysis, and Thermography in Aquatic Settings. Sci. Adv. 2019, 5, 1-14. [CrossRef]

61. Yang, Y.; Song, Y.; Bo, X.; Min, J.; Pak, O.S.; Zhu, L.; Wang, M.; Tu, J.; Kogan, A.; Zhang, H.; et al. A Laser-Engraved Wearable Sensor for Sensitive Detection of Uric Acid and Tyrosine in Sweat. Nat. Biotechnol. 2020, 38, 217-224. [CrossRef]

62. Gamella, M.; Campuzano, S.; Manso, J.; de Rivera, G.G.; López-Colino, F.; Reviejo, A.J.; Pingarrón, J.M. A Novel Non-Invasive Electrochemical Biosensing Device for in Situ Determination of the Alcohol Content in Blood by Monitoring Ethanol in Sweat. Anal. Chim. Acta 2014, 806, 1-7. [CrossRef]

63. Hubble, L.J.; Wang, J. Sensing at Your Fingertips: Glove-Based Wearable Chemical Sensors. Electroanalysis 2018, $31,428-436$. [CrossRef]

64. Bandodkar, A.J.; O’Mahony, A.M.; Ramírez, J.; Samek, I.A.; Anderson, S.M.; Windmiller, J.R.; Wang, J. Solid-State Forensic Finger Sensor for Integrated Sampling and Detection of Gunshot Residue and Explosives: Towards "Lab-on-a-Finger". Analyst 2013, 138, 5288-5295. [CrossRef]

65. Mats, d.J.; Sleegers, N.; Kim, J.; Durme, F.V.; Samyn, N.; Wang, J.; Wael, K.D. Electrochemical Fingerprint of Street Samples for Fast On-Site Screening of Cocaine in Seized Drug Powders. Chem. Sci. 2016, 7, 2364-2370. [CrossRef]

66. Cui, B.; Tertiş, M.; Cernat, A.; Sandulescu, R.; Wang, J.; Cristea, C. Finger-Based Printed Sensors Integrated on a Glove for On-Site Screening Of Pseudomonas Aeruginosa Virulence Factors. Anal. Chem. 2018, 90, 7761-7768. [CrossRef] 
67. Ciui, B.; Martin, A.; Mishra, R.K.; Nakagawa, T.; Dawkins, T.; Lyu, M.; Cristea, C.; Sandulescu, R.; Wang, J. Chemical Sensing at the Robot Fingertips: Toward Automated Taste Discrimination in Food Samples. ACS Sens. 2018, 3, 2375-2384. [CrossRef] [PubMed]

68. Islam, S.M.R.; Kwak, D.; Kabir, M.D.H.; Hossain, M.; Kwak, K.-S. The Internet of Things for Health Care: A Comprehensive Survey. IEEE Access 2015, 3, 678-708. [CrossRef]

69. Kan, C.; Chen, Y.; Leonelli, F.; Yang, H. Mobile Sensing and Network Analytics for Realizing Smart Automated Systems towards Health Internet of Things. In Proceedings of the 2015 IEEE International Conference on Automation Science and Engineering (CASE), Gothenburg, Sweden, 24 August 2015; pp. 1072-1077.

70. Spanò, E.; Di Pascoli, S.; Iannaccone, G. Low-Power Wearable ECG Monitoring System for Multiple-Patient Remote Monitoring. IEEE Sens. J. 2016, 16, 5452-5462. [CrossRef]

71. Moustafa, H.; Schooler, E.M.; Shen, G.; Kamath, S. Remote Monitoring and Medical Devices Control in EHealth. In Proceedings of the 2016 IEEE 12th International Conference on Wireless and Mobile Computing, Networking and Communications (WiMob), New York, NY, USA, 17 October 2016; pp. 1-8.

72. Chen, M.; Ma, Y.; Li, Y.; Wu, D.; Zhang, Y.; Youn, C.-H. Wearable 2.0: Enabling Human-Cloud Integration in Next Generation Healthcare Systems. IEEE Commun. Mag. 2017, 55, 54-61. [CrossRef]

73. Rahmani, A.M.; Gia, T.N.; Negash, B.; Anzanpour, A.; Azimi, I.; Jiang, M.; Liljeberg, P. Exploiting Smart E-Health Gateways at the Edge of Healthcare Internet-of-Things: A Fog Computing Approach. Future Gener. Comput. Syst. 2018, 78, 641-658. [CrossRef]

74. Farahani, B.; Firouzi, F.; Chang, V.; Badaroglu, M.; Constant, N.; Mankodiya, K. Towards Fog-Driven IoT EHealth: Promises and Challenges of IoT in Medicine and Healthcare. Future Gener. Comput. Syst. 2018, 78, 659-676. [CrossRef]

75. Almotiri, S.H.; Khan, M.A.; Alghamdi, M.A. Mobile Health (m-Health) System in the Context of IoT. In Proceedings of the 2016 IEEE 4th International Conference on Future Internet of Things and Cloud Workshops (FiCloudW), Vienna, Austria, 22 August 2016; pp. 39-42.

76. Rashed, A.; Ibrahim, A.; Adel, A.; Mourad, B.; Hatem, A.; Magdy, M.; Elgaml, N.; Khattab, A. Integrated IoT Medical Platform for Remote Healthcare and Assisted Living. In Proceedings of the 2017 Japan-Africa Conference on Electronics, Communications and Computers (JAC-ECC), Alexandria, Egypt, 18 December 2017; pp. 160-163.

77. Pang, I.; Okubo, Y.; Sturnieks, D.; Lord, S.R.; Brodie, M.A. Detection of Near Falls Using Wearable Devices: A Systematic Review. J. Geriatr. Phys. Ther. 2019, 42, 48-56. [CrossRef] [PubMed]

78. Mubashir, M.; Shao, L.; Seed, L. A Survey on Fall Detection: Principles and Approaches. Neurocomputing 2013, 100, 144-152. [CrossRef]

79. Torres, G.G.; Bayan Henriques, R.V.; Pereira, C.E.; Müller, I. An EnOcean Wearable Device with Fall Detection Algorithm Integrated with a Smart Home System. IFAC Pap. 2018, 51, 9-14. [CrossRef]

80. Wu, F.; Zhao, H.; Zhao, Y.; Zhong, H. Development of a Wearable-Sensor-Based Fall Detection System. Int. J. Telemed. Appl. 2015, 2015, 1-15. [CrossRef] [PubMed]

81. Li, Q.; Stankovic, J.A.; Hanson, M.A.; Barth, A.T.; Lach, J.; Zhou, G. Accurate, Fast Fall Detection Using Gyroscopes and Accelerometer-Derived Posture Information. In Proceedings of the 2009 Sixth International Workshop on Wearable and Implantable Body Sensor Networks, Berkeley, CA, USA, 3-5 June 2009; pp. 138-143.

82. Palmerini, L.; Klenk, J.; Becker, C.; Chiari, L. Accelerometer-Based Fall Detection Using Machine Learning: Training and Testing on Real-World Falls. Sensors 2020, 20, 6479. [CrossRef]

83. Luna-Perejón, F.; Domínguez-Morales, M.J.; Civit-Balcells, A. Wearable Fall Detector Using Recurrent Neural Networks. Sensors 2019, 19, 4885. [CrossRef]

84. Kraft, D.; Srinivasan, K.; Bieber, G. Deep Learning Based Fall Detection Algorithms for Embedded Systems, Smartwatches, and IoT Devices Using Accelerometers. Technologies 2020, 8, 72. [CrossRef]

85. Hussain, F.; Umair, M.B.; Ehatisham-ul-Haq, M.; Pires, I.M.; Valente, T.; Garcia, N.M.; Pombo, N. An Efficient Machine LearningBased Elderly Fall Detection Algorithm. arXiv 2019, 1-6. Available online: https://arxiv.org/ftp/arxiv/papers/1911/1911.11976. pdf (accessed on 26 April 2021).

86. Gunale, K.G.; Mukherji, P. Fall Detection Using K-Nearest Neighbor Classification for Patient Monitoring. In Proceedings of the 2015 International Conference on Information Processing (ICIP), Pune, India, 16-19 December 2015; pp. 520-524.

87. Pierleoni, P.; Pernini, L.; Belli, A.; Palma, L.; Valenti, S.; Paniccia, M. SVM-Based Fall Detection Method for Elderly People Using Android Low-Cost Smartphones. In Proceedings of the 2015 IEEE Sensors Applications Symposium (SAS), Zadar, Croatia, 13-15 April 2015; pp. 1-5.

88. Mazurek, P.; Morawski, R.Z. Application of Naïve Bayes Classifier in Fall Detection Systems Based on Infrared Depth Sensors. In Proceedings of the 2015 IEEE 8th International Conference on Intelligent Data Acquisition and Advanced Computing Systems: Technology and Applications (IDAACS), Warsaw, Poland, 24-26 September 2015; Volume 2, pp. 717-722.

89. Greene, S.; Thapliyal, H.; Carpenter, D. IoT-Based Fall Detection for Smart Home Environments. In Proceedings of the 2016 IEEE International Symposium on Nanoelectronic and Information Systems (iNIS), Gwalior, India, 19 December 2016 ; pp. $23-28$.

90. Pierleoni, P.; Belli, A.; Maurizi, L.; Palma, L.; Pernini, L.; Paniccia, M.; Valenti, S. A Wearable Fall Detector for Elderly People Based on AHRS and Barometric Sensor. IEEE Sens. J. 2016, 16, 6733-6744. [CrossRef] 
91. Liu, K.-C.; Yen, C.-Y.; Chang, L.-H.; Hsieh, C.-Y.; Chan, C.-T. Wearable Sensor-Based Activity Recognition for Housekeeping Task. In Proceedings of the 2017 IEEE 14th International Conference on Wearable and Implantable Body Sensor Networks (BSN), Eindhoven, The Netherlands, 9-12 May 2017; pp. 67-70.

92. Kaur, K.; Sachdeva, M. Fog Computing in IOT: An Overview of New Opportunities. In Proceedings of the ICETIT 2019: Emerging Trends in Information Technology; Singh, P.K., Panigrahi, B.K., Suryadevara, N.K., Sharma, S.K., Singh, A.P., Eds.; Springer International Publishing: Cham, Switzerland, 2020; pp. 59-68.

93. Bonomi, F.; Milito, R.; Zhu, J.; Addepalli, S. Fog Computing and Its Role in the Internet of Things. In Proceedings of the First Edition of the MCC Workshop on Mobile Cloud Computing; Association for Computing Machinery: Helsinki, Finland, 2012; pp. 13-16. 